\title{
Numerical simulation of whistler-triggered VLF emissions observed in Antarctica
}

\section{Nunn}

Department of Electronics and Computer Science, Southampton University, Southampton England

\author{
A. J. Smith \\ British Antarctic Survey, Cambridge, England
}

\begin{abstract}
The British Antarctic Survey VLF database from Halley $(L=4.3)$ and Faraday $(L=2.3)$ stations, Antarctica, has been searched for clear examples of whistler-triggered emissions (WTEs). Dominant events were the triggering of risers or quasi-constant frequency emissions from the upper arm of a whistler. A fairly frequent occurrence was the triggering of steep fallers from the whistler upper arm. At Faraday most WTE events were the triggering of long steep riscrs from the lower whistler arm. A VHS/VLF Vlasov hybrid simulation code was run and successfully simulated the main categories of WTE: risers and fallers off the upper arm and risers from the lower arm. Agreement with observations was generally very good, although in the case of triggered fallers and risers from the lower arm, very high frequency sweep rates were not obtained. The Vlasov code is highly efficient and well suited to this problem.
\end{abstract}

\section{Introduction}

This research is concerned with the nonlinear electron cyclotron resondance in the inner magnetosphere between energetic radiation belt electrons (with energies $\sim \mathrm{keV}$ ) and parallel propagating large-amplitude VLF waves. The specific topic under investigation is the triggering of VLF emissions by lightning whistlcrs. The extensive VLF database from the British Antarctic Survcy stations at Hallcy and Faraday, Antarctica, contains many terrestrial observations of VLF emissions triggered by whistlers. This database has been searched for good examples of whistler-triggered emissions (WTE's), and the main categories of event have been identified. A Vlasov plasma simulation code has been developed specifically for this problem, and the dominant types of WTE have been successfully simulated.

The phenomenon of WTEs is of considerable plasma theoretical interest and is relevant to considerations of the overall energy balance of the Earth's magnetosphere. The Vlasov numerical technique used to simulate this problem is very general and applicable to most plasma simulation problems in a collision-free regime.

Copyright 1996 by the American Geophysical Union.

Paper number 95JA03143.

0148-0227/96/95JA-03143\$05.00

\section{Overview of Nonlinear Wave Particle Interactions in the VLF Band}

Narrow-band or band-limited VLF wave energy is injected into the inner magnetosphere from the Earth from several sources. These are, in order of importance: (1) VLF radiation from lightning discharges (spherics); (2) radiation from terrestrial VLF transmitters; and (3) radiation from power line systems and from industrial plant (the so-called power line harmonic radiation). Radiation from lightning discharges is initially a broadband impulse or spheric but in the magnetosphere soon becomes quasi narrow band with the characteristic spectrographic form of the whistler, which arises from dispersive propagation through the magnetospheric plasma. VLF transmissions typically take the form of either sequences of CW pulses or close sidebands as in the frequency shift keying (FSK) or minimum shift keying (MSK) formats.

A small fraction of the energy radiated from terrestrial sources propagates through the ionosphere and enters the magnetosphere. Most of this injected VLF wave energy will be unducted with a $\mathbf{k}$ vector which becomes increasingly oblique. Although unducted radiation is ubiquitous throughout the inner magnetosphcre, it is generally of a low amplitude due to Landau damping and is not expected to produce strongly nonlinear phenomena such as triggered emissions. However, some injected VLF wave energy may be "ducted" or trapped in field-aligned enhancements of plasma density [Laird and Nunn, 1975], such that the $\mathbf{k}$ vector remains within 
a few degrees of the ambient magnetic field direction. Ducted VLF radiation would normally have relatively large amplitudes due to cyclotron resonance growth in the anisotropic electron distribution prevailing.

Ducted radiation from terrestrial VLF transmitters or lightning discharges may often reach sufficiently high amplitude levels that a number of complex, but wellknown nonlinear phenomena may occur. These include the triggering of VLF emissions [Helliwell, 1967], triggering of VLF hiss or chorus or the generation of multiple sidebands. Such phenomena are generally considered to result from nonlinear electron cyclotron resonance or phase trapping in the equatorial region. This occurs predominantly in or near the equatorial plane for two reasons. First, the cyclotron resonance velocity increases as one moves away from the equator, and thus the number of resonant particles decreases quickly. Second, the ambient field gradient increases away from the equator and eventually suppresses nonlinear trapping [Nunn, 1974].

In this paper we shall consider the observations and simulations of VLF emissions triggered by natural whistlers. Triggered emissions are a strongly and overtly nonlinear phenomenon with a relatively narrow bandwidth and are thus amenable to computational simulation. Although whistlers are also observed to trigger chorus or hiss, these phenomena have a larger bandwidth and are more difficult to simulate.

\section{Triggered VLF Emissions}

The triggering of VLF emissions is a strongly nonlinear wave particle interaction phenomenon in which ducted VLF signals "trigger" a self-sustaining VLF output, which may have a much greater amplitude and longer duration than the triggering wave. VLF emissions are usually characterized by a sweeping frequency, with a rising frequency being preferred. Fallers are fairly common with weaker signals, and hook-shaped emissions are also often observed. The final frequency of triggered emissions may be several $\mathrm{kHz}$ removed from that prevailing at the time of triggering. There is an extensive literature on this subject. The reader is particularly referred to the work of the RadioScience Laboratory at Stanford University le.g., Brice, 1963; Helliwell and Inan, 1982; Smith et al., 1985; Sá, 1990]. A very useful summary of observations, theory, and simulations of VLF emissions will be found in the work by Omura et al. [1991].

The mechanism underlying the triggering of VLF emissions is largely understood, although many puzzling problems remain. The basic theory is described by Nunn $[1974,1990]$ and Omura and Matsumoto [1982, 1985]. The review of Omura et al. [1991] provides a comprehensive review of theoretical work in this subject.

Parallel propagating VLF waves in the inner magnetosphere near the equatorial plane see a parabolic in- homogeneity due to the ambient magnetic field variation. When the VLF wave amplitude exceeds a nonlinear threshold, which is about $2 \mathrm{pT}$ at $L=4$, nonlinear electron cyclotron resonant trapping occurs, as described by Nunn [1974]. A potential well exists in $V_{z}, P$ space, where relative phase $P$ is the angle between the $V_{\perp}$ vector and the wave electric field vector. Resonant electrons in the well remain trapped and closely resonant with the wave. Parallel velocity oscillates about the local resonance velocity and $P$ oscillates about the phase trapping angle $P_{0}$, which is a function of local total inhomogeneity. When particles remain trapped for times of order of the trapping period, the resonant particle current $\mathbf{J}_{\text {res }}$ becomes nonlinear in character and dominated by trapped particles which have long interaction times with the wavefield. Two features of the nonlinear resonant particle current are significant. The first is that nonlinear growth, proportional to $-\mathbf{J}_{\text {res }} . \mathbf{E}$, is somewhat larger than the linear growth rate by a factor 2-10 say, and increases sharply with wave amplitude roughly as $E^{3 / 2}$ in a parabolic inhomogeneity. This expression applies only if the wavefield bandwidth remains much less than the trapping frequency, which is often not the case. Clearly, this large nonlinear growth provides the absolute instability necessary for a VLF emission to be self-sustaining. The second important property of $\mathbf{J}_{\text {res }}$ is that its phase is close to $P_{0}$ or $P_{0}+\pi$, where $P_{0}$ is the phase trapping angle determined by the value of local inhomogeneity [see Nunn, 1974]. Since $\mathbf{J}_{\text {res }}$ has a variable phase relative to $E_{\perp}$ that is a function of both $z$ and $t$, it is clear that the nonlinear resonant particle current can change the wavefield phase in a systematic way and is thus able to produce the sweeping frequencies observed in emissions. This concept is substantiated by Nunn [1984] which constructs numerically self-consistent models of generation regions for VLF emissions.

The triggering process is inevitably complicated and may only be properly understood by a full plasma simulation. Similarly, the dynamics of the self-sustaining generation region of a VLF emission is very complex and again requires simulation.

\section{Numerical Simulation of Triggered VLF Emissions}

Space prohibits a thorough review of all past work on the numerical simulation of triggered VLF emissions; for this, the reader is referred to Omura et al. [1991]. For reasons of computational workload all simulations of the triggered emission problem have been with one spatial dimension and three velocity dimensions, assuming propagation parallel to the ambient magnetic field. The problem is basically one of the self-consistent simulation of the interaction of a bandlimited VLF wavefield (bandwidth $\lesssim 100 \mathrm{~Hz}$ ) with a continuous, anisotropic, linearly unstable distribution of energetic electrons. In the case of triggered emis- 
sions the emission central frequency may vary by a $\mathrm{kHz}$ or more, and hence this must be a freely variable parameter. The nonlinear interaction region/simulation box extends several thousand kilometers either side of the equator and must include the region where nonlinear trapping will occur. This of course depends upon the maximum wave amplitudes achieved in the ensuing simulation.

Several simulations of the triggered emission problem have been undertaken, all with triggering by $\mathrm{CW}$ pulses such as from terrestrial VLF transmitters or from experimental facilities such as Siple Station, Antarctica [e.g., Carlson et al., 1990]. Omura and Matsumoto [1982, 1985] performed large particle-in-cell (PIC) simulations of VLF emission triggering and achieved interesting results. However, the use of PIC codes presents difficulties, sincc cyclotron resonance vclocity is a strong function of position and time. To track a VLF emission over a large frequency range requires a huge number of simulation particles.

Simulations of triggered VLF emissions have been performed by Nunn [1990, 1993] using the Vlasov hybrid simulation (VHS) technique. This technique will be used for simulations of WTEs in this paper. It will be described in detail in a later section of this paper, but essentially it uses a simulation box in phase space in which the parallel velocity coordinate range is matched to the local resonance velocity, in both space and time. The code monitors only resonant or nearly resonant particles, no matter how the frequency develops in time and space. Nunn [1990] applied this technique to the simulation of emissions triggered by $\mathrm{CW}$ pulses from the Siple transmitter at $4.2 \mathrm{kHz}$ and also from NAA at $14.7 \mathrm{kHz}$. Generally, the code was very successful at triggering rising frequency emissions, and the numerical triggering process was very repeatable and stable. Using low growth rates and/or distribution functions that are isotropic at high pitch angles, triggered fallers have now been obtained from this code.

The aim of this rescarch is to upgrade substantially and modify the VHS/VLF code so as to simulate WTEs and to compare the results closely with observations. Simulation of WTEs has not been done before and is considerably more difficult than simulating emissions triggered by CW pulses. This is because of the large bandwidth of the triggering signal.

Before discussing the data, two important aspects of the simulation need to be mentioned. Although simulated in one spatial dimension the real problem of nonlinear wave-particle interactions (WPI) for VLF waves trapped in a duct is inherently three dimensional (3D). The reason is that the nonlinear resonant particle current generated in the duct radiates into unducted modes giving a substantial nonlinear unducting loss which cuts in when the wave amplitude reaches nonlinear levels. The purely $1 \mathrm{D}$ simulation is absolutely unstable at nonlinear amplitudes, and there is no adequate saturation mechanism operating. Accordingly, in 1D codes it is necessary to model the nonlinear unducting loss phe- nomenologically. This is not ideal but quite unavoidable. Eventually, computer technology will permit full 3D simulations.

The second matter concerns the perpendicular velocity coordinate. Low pitch angle particles are linear, and there are few high pitch angle particles. We are mainly concerned with particles with pitch angles in the range $40^{\circ}-70^{\circ}$, and the contribution to $\mathbf{J}_{\text {res }}$ as a function of $V_{\perp}$ will be sharply peaked. In the simulation, relatively few grid points are required in the coordinate $V_{\perp}$. Indeed with VHS codes, simulations may be run with only one value of $V_{\perp}$, and the results will look little different from simulations with many $V_{\perp}$ grid points. Note that with particle-in-cell codes it is not permissible to do this, as explained by Nunn [1990]. However, the case where the sign of the contribution to $\mathbf{J}_{\text {res }}$ changes with $V_{\perp}$ will require more $V_{\perp}$ grid points. Therc is rcason to believe that this may often be the case, in which case a $V_{\perp}$ grid of some 3-10 points would be called for.

\section{VLF Database}

British Antarctic Survey has collected VLF broadband $(0.5-10 \mathrm{kHz})$ goniometer data from its research stations at Halley $\left(76^{\circ} \mathrm{S}, 26^{\circ} \mathrm{W}\right)$ and Faraday $\left(65^{\circ} \mathrm{S}\right.$, $64^{\circ} \mathrm{W}$ ) since 1967 . The antennae are two orthogonal vertical loops, orientated north-south and east-west, sensitive to the horizontal magnetic components of the wavefield $B_{x}$ and $B_{y}$ [Yearby and Smith, 1994; Smith, 1995]. The data are archived on magnetic tape (analogue tape until 1993; digital audio tape from 1994).

Since 1983 the data have been analyzed by a powerful interactive signal processing tool Advanced VLF Data Analysis System (AVDAS) which digitises (after antialias filtering), Fourier transforms and displays the data in spectrographic form at the real-time playback speed (two channels digitized at up to a $40-\mathrm{kHz}$ rate, that is, a $20-\mathrm{kHz}$ bandwidth). The original Mk1 8-bit system [Smith and Yearby, 1987], has since 1990 been superseded by a modern 12-bit version, developed by High Greave Associates of Sheffield, England, and based on digital signal processing (DSP) technology [Smith et al., 1994].

The data stream may be analyzed in the bands 0$1 \mathrm{kHz}, 0-2 \mathrm{kHz}, 0-5 \mathrm{kHz}$, or $0-10 \mathrm{kHz}$. Fast Fourier transform (FFT) size is selectable at either 256,512 , or 1024 , with a redundancy factor $r$ of 1,2 , or 4 , and Hanning weighting. AVDAS also has a feature whereby successive spectra may be averaged, though this facility is not appropriate in the present case. The output of each FFT is presented as a vertical strip on a display screen with a colour coding adjustable by the operator, resulting in a constantly updated spectrogram; examples (with a grey-scale) are shown in Figure 1. Typical analysis parameters might be frequency range $f=10 \mathrm{kHz}$, FFT transform size $N=1024$, redundancy $r=2$, giving a frequency resolution of $25 \mathrm{~Hz}$ and a time resolution of $20 \mathrm{~ms}$.

A valuable facility for interactive whistler analysis 


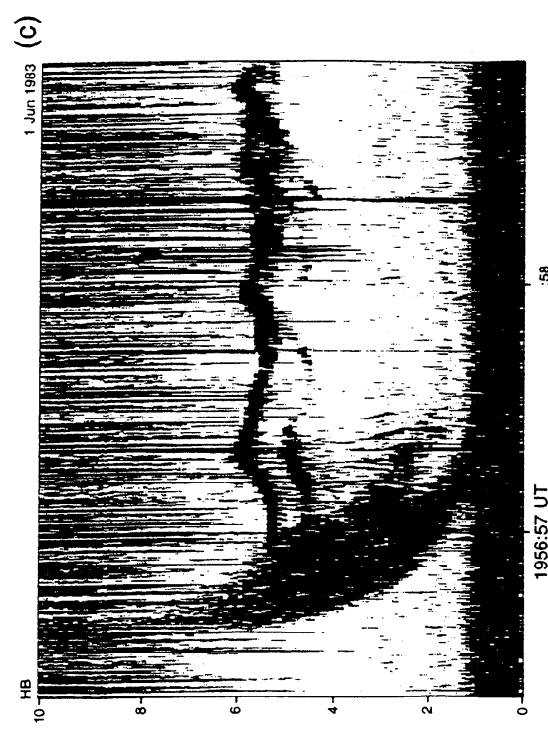

을

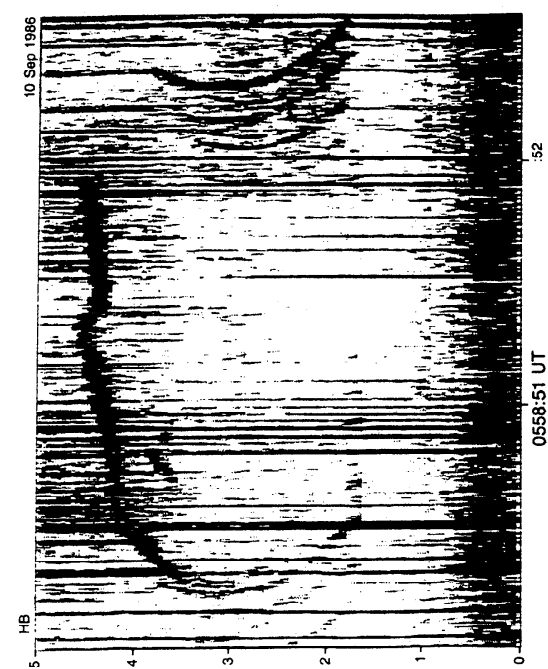

(્)

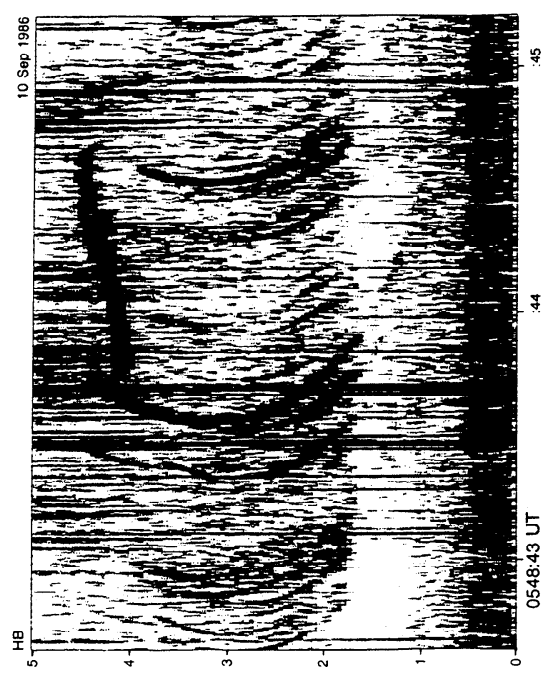

(zHy) $f$

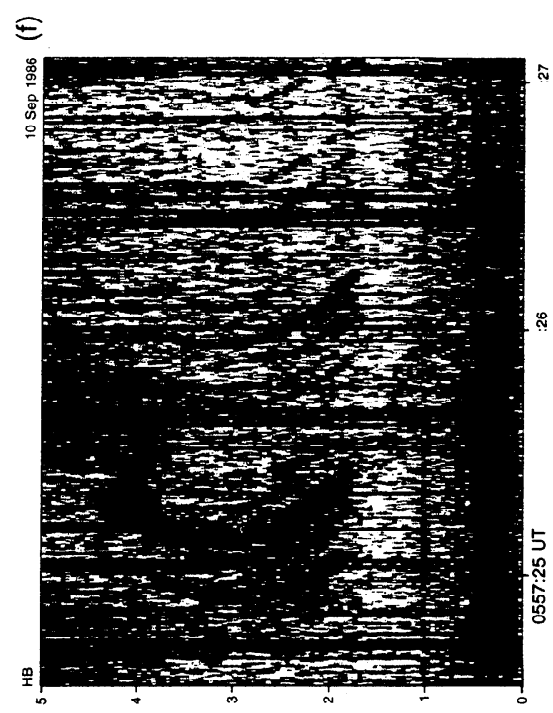

(્)

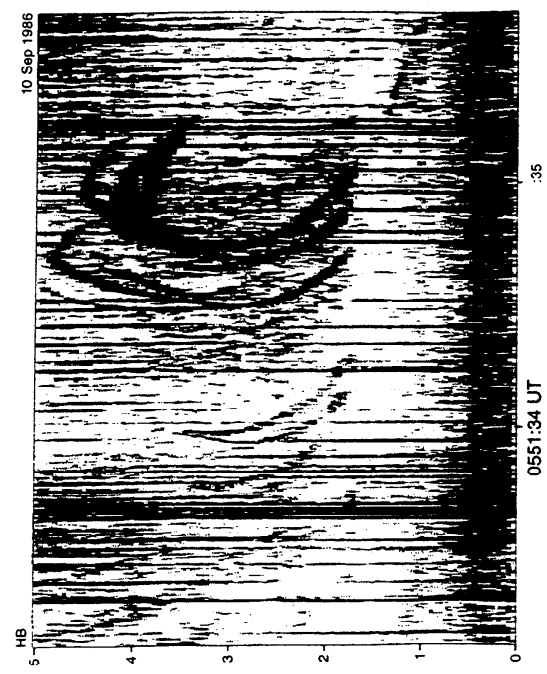

으

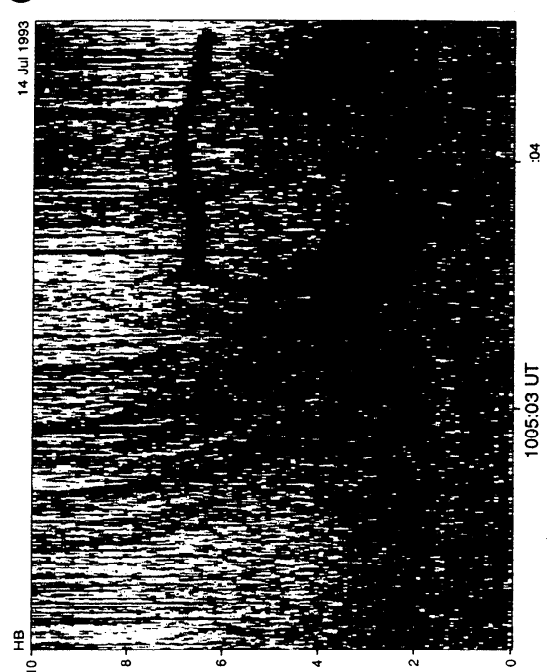

$(z H x) f$

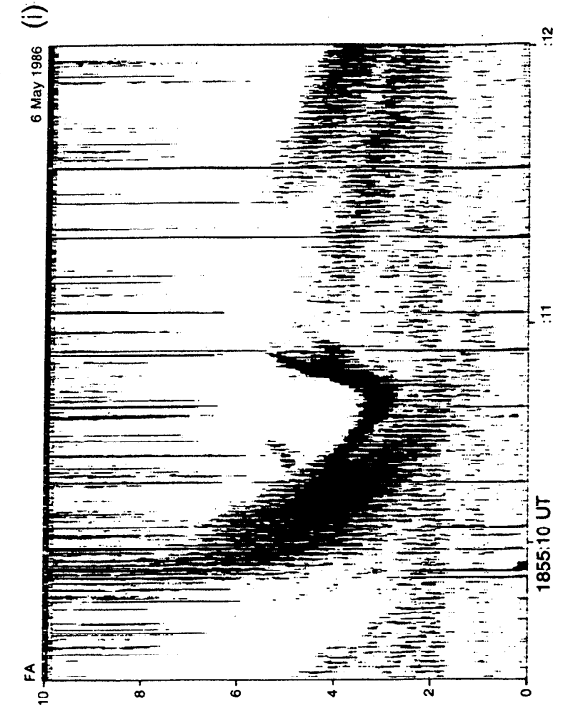

$\stackrel{\dot{f}}{5}$

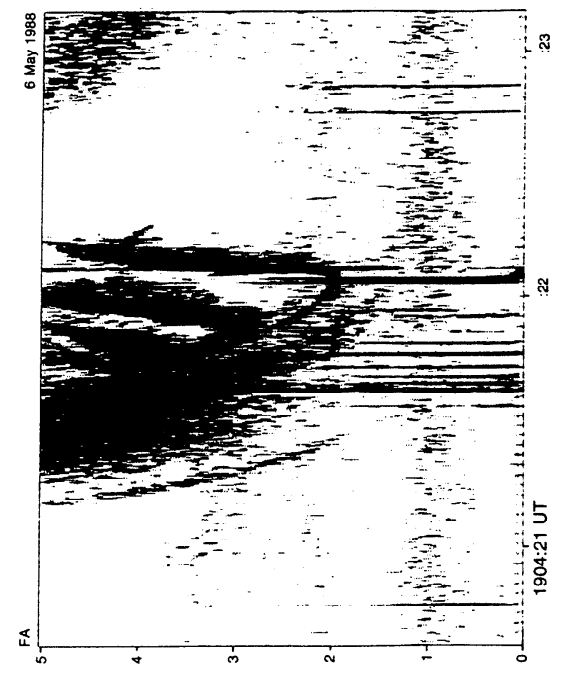

O)

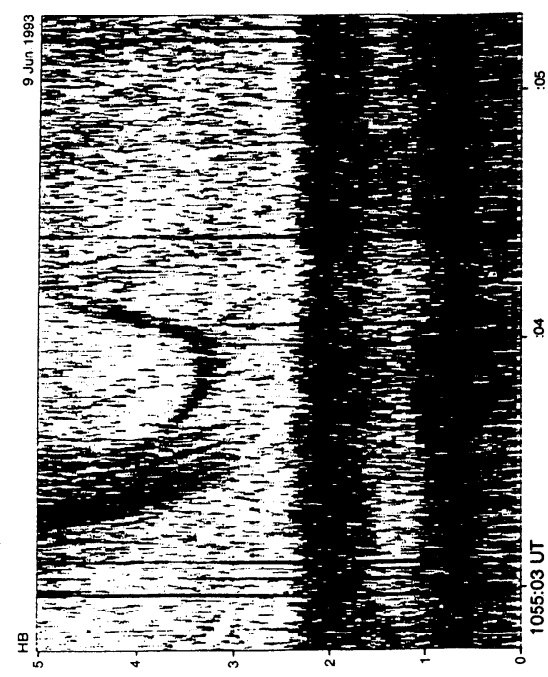

(zHY) $f$ 
Table 1. Summary of Data for Selected Examples of WTEs

\begin{tabular}{rrrrrrrrrrrrrr}
\hline$N$ & Station & Year & Day & UT & $N_{\text {FFT }}$ & $r$ & $\delta f$ & $\delta \iota$ & Type & $\Delta f$ & $\tau$ & $L$ & $d f / d \iota$ \\
\hline 1 & HB & 1986 & 253 & $05: 48$ & 256 & 4 & 50 & 5 & Riser & 160 & 1.0 & 4.70 & 400 \\
2 & HB & 1986 & 253 & $05: 58$ & 256 & 4 & 50 & 5 & Riser & 140 & 1.5 & 4.75 & 300 \\
3 & HB & 1983 & 152 & $19: 56$ & 256 & 2 & 100 & 5 & QCFE & 250 & 2.0 & 3.64 & 0 \\
4 & HB & 1993 & 195 & $10: 05$ & 256 & 2 & 100 & 5 & QCFE & 200 & 1.2 & 4.10 & 0 \\
5 & HB & 1986 & 253 & $05: 51$ & 256 & 2 & 50 & 5 & Faller & 80 & 0.4 & 4.68 & -1500 \\
6 & HB & 1986 & 253 & $05: 57$ & 256 & 4 & 50 & 5 & Faller & 100 & 0.5 & 4.70 & -800 \\
7 & HB & 1993 & 160 & $10: 55$ & 256 & 4 & 50 & 5 & Hook & 140 & 0.7 & 3.50 & \pm 2000 \\
8 & FA & 1988 & 127 & $19: 04$ & 256 & 4 & 50 & 5 & Riser & 250 & 0.5 & 3.20 & 3000 \\
9 & FA & 1988 & 127 & $18: 55$ & 256 & 2 & 100 & 5 & Hook & 250 & 0.4 & 3.10 & 3000 \\
\hline
\end{tabular}

For each of the nine events $(N)$ illustrated in Figure 1, the table shows the station (HB, Halley; FA, Faraday) and date/time of the observation; the FFT transform size, redundancy, frequency resolution (hertz) and time resolution (milliseconds) of the AVDAS spectrogram plot presented; the type of WTE; and its approximate bandwidth (hertz), duration (seconds), $L$ shell and frequency sweep rate $\left(\mathrm{Hz} \mathrm{s}^{-1}\right)$.

is the SCALE function. Using a screen cursor and a mouse, the operator clicks 3 times on a whistler trace at well-separated points and the software then estimates the $L$ shell appropriate to the magnetospheric path for that whistler. Also calculated are equatorial electron density, whistler nose frequency $f_{n}$, and the nose delay $t_{n}$. The calculations use the expressions found in the work by Ho and Bernard [1973], which arc based upon a dipole magnetic field model and a diffusive equilibrium model of cold plasma density. Best results are obtained when the whistler trace is sharp, continuous, and welldefined and extends over a wide frequency range; in less favorable cases, the random errors may be reduced somewhat by averaging the results of repeated analysis of the same whistler [Smith et al., 1975; Sazhin et al., 1992].

Searching for WTE events in the data can be quite time-consuming, as interesting events occur in groups at widely separated times. As an invaluable aid to the search procedure, AVDAS may be configured to produce "quicklook" summary plots of the VLF data in highly compressed spectrographic form (for an example, see Figure 5 of Smith [1995]) enabling time spans rich in triggering events to be pinpointed.

\section{Selected Examples of Whistler- Triggered Emissions}

The BAS VLF database from Halley and Faraday has been searched for WTEs for the years 1983, 1986, 1988, and 1993, the search being concentrated during periods of known high activity. Good examples are quite hard to find, but fortunately they tend to congregate during short periods when conditions are very favorable. More occurrences were found at Halley $(L \simeq 4.2)$ than at the lower-latitude Faraday $(L \simeq 2.5)$. The reason for this is that at high $L$, resonant energies are lower, and the allowable size of the nonlinear cmission generation region is larger.

In this paper we shall present nine examples of WTEs; seven from Halley and two from Faraday. Data appertaining to these observations are summarized in Table 1. Estimates of the $L$ value and nose frequency for the causative whistler are obtained from the AVDAS SCALE facility. WTEs at Halley may be divided into several quite distinct types. The most common is the triggering of a "slow" riser from above the nose frequency, often from the top of the visible whistler trace. The first example in Figure 1a shows a slow riser originating from just below the top of the whistler. The $L$ value for this whistler is about 4.7 , and the equatorial electron density about $200 \mathrm{~cm}^{-3}$. The emission lasts about $1 \mathrm{~s}$ and has a bandwidth of order $150 \mathrm{~Hz}$ and a sweep rate of about $400 \mathrm{~Hz} \mathrm{~s}^{-1}$. These are very typical values. It should be noted that these observations are terrestrial, and the dispersion between the equatorial generation region and the ground needs to be taken into account. The true value of $d f / d t$ at the equator would be approximately $500 \mathrm{~Hz} \mathrm{~s}^{-1}$. Figure $1 \mathrm{~b}$ shows a long enduring slow riser triggered from the top of a

Figure 1. (opposite) AVDAS spectrograms showing examples of different types of WTEs observed at Halley (a)-(g) and Farady (h)-(i), and listed in Table 1. (a) Event 1: a slow riser triggered from the top or near the top of a whistler. (b) Event 2: another example of a slow riser triggered from the top of a whistler. (c) Event 3: an irregular long-enduring QCFE. (d) Event 4: a QCFE triggered at the top of a whistler. (e) Event 5: multiple steep fallers triggered from the top of whistlers. (f) Event 6: a single slow faller triggered from the top of a whistler. (g) Event 7: an upward hook triggered from the lower arm of a whistler. (h) Event 8: strong steep risers triggered from the lower arm of a whistler. (i) Event 9: upward hook triggered at the bottom of a whistler. 
weak whistler at $L=4.75$ on the same day. Duration is $1.5 \mathrm{~s}$, and slope is about $300 \mathrm{~Hz} \mathrm{~s}{ }^{-1}$.

The second clearly definable category of WTE is that of the long quasi-constant frequency emission (QCFE), or "quasi-constant tone" in the nomenclature of Helliwell [1965]. Figure 1c shows a very long enduring emission ( $\sim 2 \mathrm{~s})$ triggered by a diffuse whistler at a rather lower $L$ shell of $L=3.64$. The value of sweep rate $d f / d t$ fluctuates a little and shows discontinuous behavior, probably associated either with sudden changes in particle flux or nonlinear interaction with strong unducted VLF signals. Figure 1d also shows another good example of a QCFE, triggered near $L=4.1$. The emission, which lasts about $1.2 \mathrm{~s}$, eventually becomes a slow faller with a sweep rate in the region of $-900 \mathrm{~Hz} \mathrm{~s}^{-1}$.

A category of event which is fairly common is that of the triggering of steep fallers from the top of the observable whistler trace. Several typical examples, which originate from an $L$ value of $\sim 4.68$ are shown in Figure 1e. These fallers are short, of order 0.4-s duration, and have sweep rates of order $-1.5 \mathrm{kHz} \mathrm{s}^{-1}$. These events are analogous to the stecp fallers observed to be triggered at the end of short $\mathrm{CW}$ pulses radiated from the Siple VLF transmitter [Helliwell, 1983]. It should be noted that the sweep rates of these emissions at the equator would be rather less in magnitude, about $-1 \mathrm{kHz} \mathrm{s}^{-1}$, due to dispersion. These short steep fallers often have a narrower bandwidth than the slow risers, of order $80 \mathrm{~Hz}$ here, and appear to be associated with low linear growth rates and fairly weak triggering signals. Another example of a faller triggered from the top of a whistler is shown in Figure 1f. This emission was triggered at an $L$ value of 4.69 and has a more moderate sweep rate of about $-1.0 \mathrm{kHz} \mathrm{s}^{-1}$.

Triggering from the lower branch of a whistler is not commonly observed at the geomagnetic latitude of Halley. However in Figure $1 \mathrm{~g}$ we present one example of an upward hook triggered from the bottom of a whistler. Not surprisingly, the causative whistler was from a much lower $L$ shell, about $L=3.5$. The emission has many of the characteristics of lower $L$ shell triggering, such as a much steeper sweep rate of order $2 \mathrm{kHz} \mathrm{s}^{-1}$.

Triggered emissions observed at Faraday are rarer than at Halley and tend to be exclusively steep risers triggered from the lower part of the observable whistler trace. Figure $1 \mathrm{~h}$ shows a typical example originating from $L=3.5$. The sweep rate $d f / d t$ is very steep indeed, but when dispersion is removed the sweep rate at the equator is in the region of $3 \mathrm{kHz} \mathrm{s}^{-1}$. A not uncommon emission type at low $L$ shells is the upward hook triggered from the lower whistler branch. Figure $1 \mathrm{i}$ shows an example of this type. The $L$ value for the triggering whistler is not easily obtained in this case; it is probably about $L=3.1$.

Finally, it should be noted that the BAS VLF database contains a large number of triggering and nonlinear WPI events that are of considerable interest in their own right, but for which considerations of space prevents their inclusion in this paper. Such events are (1) Triggering of a profusion of risers from one whistler in a very active situation. These may originate from all parts of the whistler, including "prompt triggering" from the nose; (2) triggering of hiss bands from the upper part of a whistler; (3) triggering of chorus from the upper part of a whistler; and (4) triggering of strong risers from the top of a hiss band. Investigation and simulation of these events will be the subject of future research.

\section{Description of the VHS/VLF Code}

In this section we will describe the essential features of the VHS/VLF code. We first assume band-limited parallel propagating VLF waves with $\mathbf{k} \| \mathbf{B}_{\mathbf{0}}$. The linear dispersion relation is

$$
n^{2}=\frac{k^{2} c^{2}}{\omega^{2}}=\frac{\Pi_{\mathrm{e}}^{2}}{\omega(\Omega-\omega)}
$$

where $\Pi_{e}$ is the local plasma frequency and $\Omega$ is the local electron gyrofrequency. The displacement current has been neglected in the inner magnetosphere. In what follows we shall employ a system of dimensionless variables in which the unit of time is

$$
\bar{t} \equiv \frac{1}{\bar{\omega}}=\frac{2}{\Omega(0)}
$$

and the unit of distance is

$$
\bar{z} \equiv \frac{1}{\bar{k}}=\frac{c}{\Pi_{\mathrm{e}}(0)}
$$

In the coordinate system used, $z$ is along the field line, positive in the wave propagation direction, with the equator at $z=0$; the coordinate directions $x$ and $y$ are orthogonal to $z$. The ambient magnetic field is assumed to have a parabolic dependence upon $z$ in the equatorial zone, that is, in dimensionless units we have

$$
\beta=1+\frac{1}{2} \chi z^{2}=\Omega(z) / \Omega(0)
$$

where

$$
\chi=\left(9 \times 10^{-14}\right) /(L \cdot 6370 \bar{k})^{2}
$$

The cold plasma density $N_{\mathrm{e}}(z)$ is assumcd also to have a parabolic dependence upon $z$,

$$
\gamma_{\mathbf{e}} \equiv N_{\mathrm{e}}(z) / N_{\mathbf{e}}(0)=1+\frac{1}{2} \nu \chi z^{2}
$$

The actual dependence of $N_{\mathrm{e}}$ on $z$ is not critical for this problem. In most of the simulation runs we shall use $\nu=0.3$.

The dispersion relation now becomes

$$
k^{2}=\frac{\gamma_{\mathrm{e}} \omega}{2 \beta-\omega}
$$

where $\omega$ and $k$ are dimensionless as defined in (2) and (3). 
The $z$ coordinate range for the simulation box is fixed at $z=z_{l} \rightarrow z_{r}$ (typically several thousand kilometers on either side of the equator). This range of $z$ values covers the region where nonlinear trapping may occur for the saturation wave amplitude $B_{\max }$.

Since the simulation is band limited, we first define a base frequency $\omega_{0}$, and a base wave number $k_{0}(z)$. Define the base phase $\Phi$ by

$$
\Phi=\omega_{0} t-\int^{z} k_{0}\left(z^{\prime}\right) d z^{\prime}
$$

A dimensionless complex amplitude $\tilde{R}$ may be defined as

$$
\tilde{R} \equiv R \mathrm{e}^{j \phi}=\left(\frac{e \bar{k}}{m \bar{\omega}^{2}}\right) \mathcal{E}
$$

where

$$
\mathcal{E}=E_{\perp} \mathrm{e}^{-j \Phi} ; E_{\perp}=E_{x}+j E_{y}
$$

Similarly, the dimensionless complex resonant particle current is given by

$$
\tilde{J}=\left(\frac{e \mu_{0}}{2 m \bar{\omega} \bar{k}}\right) \mathcal{T}
$$

where

$$
\mathcal{J}=J_{\perp} \mathrm{e}^{-j \Phi} ; J_{\perp}=J_{x}+j J_{y}
$$

Under the narrowband approximation, $\tilde{R}$ and $\tilde{J}$ are slowly varying functions of $z$ and $t$, and with this approximation, the dimensionless field push equation becomes very simply

$$
\left\{\frac{\partial}{\partial t}+V_{y} \frac{\partial}{\partial z}\right\} \tilde{R}--\frac{\omega_{0} V_{g}(0)}{k_{0}} \tilde{J}-\gamma(R) \tilde{R}
$$

where $V_{g}(z)$ is the dimensionless group velocity given by

$$
V_{g}(z)=\omega_{0}\left(2 \beta-\omega_{0}\right) / k_{0} \beta
$$

In the WTE problem the average frequency in the simulation box may vary widely with time. In this case it is necessary to reset periodically the base frequency and redefine $\tilde{R}, \tilde{J}$ and all particle variables. The nonlinear damping term $-\gamma \tilde{R}$ represents nonlinear unducting loss which is very large when the wave-particle interaction becomes strongly nonlinear at amplitudes of $\sim 4-5 \mathrm{pT}$. Nonlinear damping is presumed to increase rapidly above this level, thus giving an effective saturation mechanism and a maximum wave amplitude level $B_{\max }$.

Across the domain $z=z_{l} \rightarrow z_{r}$ two spatial grids are defined. Simulation particles (SP's) are defined on the "particle grid," which has $N_{j}=512$ or 1024 grid points. Since all particles move with a parallel velocity close to the cyclotron resonance velocity $V_{\text {res }}$ where, in dimensionless units

$$
V_{\text {res }}(z)=\left(\omega_{0}-2 \beta\right) / k_{0}
$$

at each time step, all particles move one grid point to the left on the particle grid (a distance of $\left|V_{\text {res }}(0)\right| \Delta t$ ). The complex field $\tilde{R}$ is defined on the "field grid" which has $N_{r}$ grid points where $N_{r}=N_{j} \cdot\left|V_{\text {res }}(0)\right| / V_{g}(0)$. At each time step the field values $\tilde{R}$ are advected one grid point to the right, a distance of $V_{g}(0) \Delta t$. In order to implement particle push and field push equations (18) and (13) it is necessary to cross interpolate between these two grids using precomputed coefficients.

\section{Particle Equations of Motion}

The simulations in this paper use $N_{V_{\perp}}=1$ or 3 , the valucs being chosen to correspond to those pitch angles at which the contribution to resonant particle current $\tilde{J}$ is maximum (typically in the range $45^{\circ}-65^{\circ}$ ). Since the total change in $V_{\perp}$ for a resonant particle will be small in the interaction region, to a good approximation $V_{\perp}$ may be put constant where it occurs in the equations of motion (equations (18), (19), and (20)), and in expressions for $\tilde{J}$ (equations (25) and (26)).

The equations of motion of cyclotron resonant particles are written in terms of variables $\psi$ and $V^{*}$. The variable $\psi$ is the phase of the perpendicular velocity vector relative to base phase $\Phi$, that is,

$$
\psi=L \tilde{V}_{\perp}-\Phi ; \tilde{V}_{\perp}=V_{x}+j V_{y}
$$

and $V^{*}$ is defined by

$$
V^{*}=V_{z}-V_{\mathrm{res}}(z)
$$

It may be shown that the dimensionless particle equar tions of motion are [Nunn, 1990]

$$
\begin{aligned}
\dot{\psi} & =k_{0} V^{*} \\
\dot{V}^{*} & =-\frac{R k_{0} V_{\perp}}{\omega_{0}} \cos (\psi-\phi)+Q_{0}(z)
\end{aligned}
$$

where $Q_{0}(z)$ is the net inhomogeneity given by

$$
Q_{0}(z)=\chi z\left(\frac{3 V_{\mathrm{res}}}{k_{0}}-\frac{V_{\perp}^{2}}{2 \beta}\right)+\frac{\nu \chi z V_{\mathrm{res}}^{2}}{2 \gamma_{e}} .
$$

The resonant particle current $\tilde{J}$ is computed from the quantity $\Delta W$, the integrated energy change of a particle. The ratc of change of $\Delta W$ is given by

$$
\Delta \dot{W}=-R V_{\perp} \cos (\psi-\phi)
$$

and the rate of change of particle magnetic moment $\Delta \dot{\mu}$ is given by

$$
\Delta \dot{\mu}=\left(2 / \omega_{0}\right) \Delta \dot{W}
$$

where

$$
\mu=V_{\perp}^{2} / 2 \beta
$$

For each value of $V_{\perp}^{l}$, and for each point on the particle grid $z_{j}$, a two-dimensional space in the coordinates $V^{*}$ and $\psi$ is defined. There are thus $N_{j} \cdot N_{V_{\perp}}$ such spaces, and the VHS procedure is applied to each separately. The range of the variable $V^{*}$ is $V_{1}^{*} \rightarrow V_{2}^{*}$ which in- 
cludes the range of resonant vclocitics appertaining to the global wavefield spectrum and extends three nonlinear resonant trapping widths (appropriate to $R_{\max }$ ) on either side [see Nunn, 1990]. In the original version of the code, $V_{1}^{*}$ and $V_{2}^{*}$ were the same for all $\left(\psi, V^{*}\right)$ planes. It was found that $N_{V^{*}}=40$ grid points in the $V^{*}$ coordinate and $N_{\psi}=20$ in phase gave sufficient resolution in velocity space.

At time $t=0$, each $\left(\psi, V^{*}\right) 2 \mathrm{D}$ plane is evenly filled with simulation particles at a density of $1.2 /$ grid square, or about 800 SPs in total. At each time step the particles are pushed using equations of motion (18) and (13), discretised to second order accuracy, and the entire assembly of particles is transferred one spatial grid point to the left. According to the VHS formalism [Nunn, 1993], at each time step the quantity $\Delta W$ is interpolated from the SP's onto the fixed $\left(\psi, V^{*}\right)$ grid. For grid point $i j$ wo have

$$
\Delta W_{i j}=\left\{\sum_{l} \Delta W^{l} \alpha_{l}\right\} /\left\{\sum_{l} \alpha_{l}\right\}
$$

where the sum $l$ is over all particles in the neighboring four-square area, each with value $\Delta W^{l}$. The $\alpha_{l}$ are area weighting factors as used in PIC codes. Since particle density in phase space is conscrved according to Liouville's theorem, very few grid points $(<1 \%)$ are void and have no particles in the neighboring four-square area. Where this happens, a value of $\Delta W_{i j}$ may be found by interpolating from neighboring grid points. Note that grid points near the boundary in variable $V^{*}$ are often "void," and these boundary grid points are not used in the calculation of resonant particle current.

\section{Calculation of Resonant Particle Current $\tilde{J}$}

With $\Delta W$ defined on a $2 \mathrm{D}$ grid in $\left(\psi, V^{*}\right)$ space at all values of $z_{j}$ and all values of $V_{\perp}^{l}$ in the simulation, the resonant particle current $\tilde{J}$ may be readily calculated. The current is given by a summation of contributions $J_{s}$ from each pitch angle beam in the simulation, that is,

$$
\tilde{J}(z, t)=C \sum_{l=1}^{N_{V_{\perp}}} J_{s}\left(z, t, V_{\perp}^{l}\right)
$$

Employing Liouville's theorem, $J_{s}$ may be expressed as an integral over the $\left(\psi, V^{*}\right)$ plane as follows

$$
\begin{aligned}
J_{s}\left(z, t, V_{\perp}\right)= & \int_{0}^{2 \pi} \int_{V_{1}^{*}}^{V_{2}^{*}}\left\{F_{0}(\mu, W)-F_{0}\left(\mu-\frac{2}{\omega_{0}} \Delta W\right.\right. \\
& W-\Delta W)\} \cdot V_{\perp}^{2} \mathrm{e}^{j \psi} d \psi d V^{*}
\end{aligned}
$$

where the quantity $F_{0}(\mu, W)$ is the static unperturbed distribution function, energy $W$ is given by

$$
W=V_{\perp}^{2} / 2+\left(V_{\text {res }}+V^{*}\right)^{2} / 2
$$

and magnetic moment $\mu$ is given by (22).

It should be noted that the computation of current $\tilde{J}$ in the code may employ any function $F_{0}$, no matter how
Table 2. Data for Unperturbed Distribution Function

\begin{tabular}{rrrr}
\hline$n$ & $K_{n}$ & $T_{\perp}, \mathrm{eV}$ & $T_{\|}, \mathrm{eV}$ \\
\hline 1 & 1722.0 & 25 & 25 \\
2 & 5.8 & 300 & 150 \\
3 & 0.034 & 2000 & 1000 \\
4 & 0.0006 & 10000 & 5000 \\
5 & 0.00003 & 10000 & 20000 \\
\hline
\end{tabular}

complex, as the program first creates a lookup table for $F_{0}$. There is no requirement that $\Delta W$ be small, and $F_{0}$ is allowed to have steplike discontinuities. The code evaluates $J_{s}$ at each position $z_{j}$ and for each value of $V_{\perp}^{l}$ by a discrete summation over the grid points in the $\left(\psi, V^{*}\right)$ plane.

In these computations, $F_{0}(\mu, W)$ is modeled by five superimposed bi-Maxwellian functions as follows:

$$
F_{0}(\mu, W)=\sum_{n=1}^{5} K_{n} \mathrm{e}^{-\mu / T_{\perp}^{n}} \mathrm{e}^{-(W-\mu) / T_{\|}^{n}}
$$

where the $T_{\perp}^{n}$ are the perpendicular temperatures and $T_{\|}^{n}$ are the parallel temperatures for the $n$th component. The constants $K_{n}, T_{\perp}^{n}$ and $T_{\|}^{n}$ are taken from a paper by Thorne and Horne [1994] and shown in Table 2. Figure 2 plots contours of $F_{0}(\mu, W)$ in the $V_{z}, V_{\perp}$ plane at the equator. Contours are plotted at equal intervals. This model fits observed particle fluxes on OGO 3. An anisotropy factor of 1 was used and found to give strong triggered emissions in the simulations. In (24) the quantity $C$ is a global constant adjusted to bring the linear cyclotron growth rate in the simulation to the desired level.

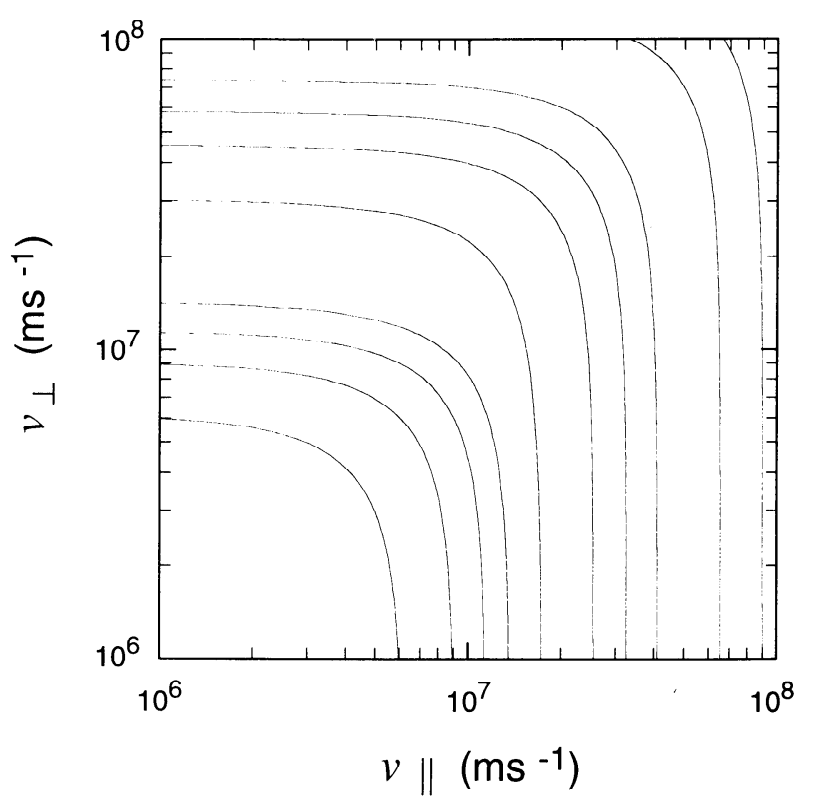

Figure 2. Contour plot of $F_{0}(\mu, W)$ in the $V_{z}, V_{\perp}$ plane at the equator. 


\section{Field Control}

At each time step $t_{n}$ the wavefield $\tilde{R}\left(t_{n}, z_{j}\right)$ is spatially DFTd, and the center wave number and equivalent frequency $f$ determined. The wavefield is then bandpass filtered by DFT/IDFT to the frequency range $f-f_{\mathrm{bw}} \rightarrow f+f_{\mathrm{bw}}$. In the original version of the program the passband was a global quantity with a width $2 f_{\mathrm{bw}}$ of about $100 \mathrm{~Hz}$. When the global average frequency is more than $10 \mathrm{~Hz}$ from the base frequency, the base frequency must be reset. This is purely a matter of numerical housekeeping and does not give rise to discontinuities in output variables. At every time step the $V^{*}$ range $V_{1}^{*} \rightarrow V_{2}^{*}$ of the phase box is adjusted to be centred on the resonance velocity of the centre frequency of the current global wavefield spectrum. The filtering operation on $\tilde{R}$ serves to keep the simulation stable and confine the wavefield spectrum to a frequency range for which the code is able to provide resonant particles.

\section{Particle Control}

The VHS formalism requires particles leaving the current phase box to be discarded. Thus particles leaving the range $V_{1}^{*} \rightarrow V_{2}^{*}$ are removed from the simulation since they are non resonant with the current global wavefield. Where phase fluid is flowing into the $\left(\psi, V^{*}\right)$ box new particles must be created. This is done by searching boundary cells at $V^{*}=V_{1}^{*}$ and $V_{2}^{*}$. If particles are not present, now particles are inserted into the phase fluid with a value of $\Delta W$ derived from linear theory. In the code more complex controls are in place to ensure that the overall density of SPs in phase space does not exceed about 1.2 per grid cell.

\section{Computational Aspects}

The code currently operatcs with about half a million particles and takes about 6 hours to run on a Cray YMP and 12 hours on a SUN SPARC server. The code is fully vectorised and optimised on the Cray YMP and eligible for implementation on massively parallel machines.

\section{Code Output}

The following graphical simulation outputs are available: (1) Plots of field amplitude $R$ and field additional phase $\phi$, as functions of $z$. (2) The component of $\tilde{J}$ parallel to $E_{\perp}$, termed $J_{r}$, as a function of $z$. (3) The component of $\tilde{J}$ perpendicular to $E_{\perp}$, termed $J_{i}$, as a function of $z$. (4) The field $\tilde{R}$ is spatially DFT'd with Hamming weighting. The spectra are displayed with the wavenumber axis (converted to equivalent frequency $\omega \prime)$ vertical, and successive spectra are stacked horizontally to give a $f-t$ spectrogram plot. The equivalent frequency $\omega / / 2 \pi$ is given by

$$
\omega \prime=\bar{\omega}\left(\omega_{0}+V_{g} \Delta k\right)
$$

where $V_{g}$ is dimensionless group velocity, $\omega_{0}$ is dimensionless base frequency and $\Delta k$ is the dimensionless wave number component of $\tilde{R}(z)$. In the absence of resonant particle current each component of $\tilde{R}$ with wave number $\Delta k$ would present to a stationary observer as a frequency $\omega /$. The $f-t$ spectrogram gives a good overview of the progress of the simulation but at a very high frequency resolution $(\sim 1 \mathrm{~Hz})$ compared to AVD $\Lambda \mathrm{S}$ plots and with a correspondingly low effective time resolution. (5) Another useful form of output is the $f-t$ plot for the wavefield data stream emerging from the right hand side of the simulation box. These data are block DFT'd with Hamming weightings and high redundancy factors of $\sim 10$. The resultant spectrogram has frequency resolutions down to $15 \mathrm{~Hz}$. It has not been possible to present the output data stream with AVDAS frequency resolutions of 50 or $100 \mathrm{~Hz}$ and time resolutions of $\sim 20-10 \mathrm{~ms}$ because of the narrow bandwidth of the simulation and limitations on the number of spatial grid points due to run time considerations. This makes very precise comparison with AVDAS data difficult.

\section{Updates to the VHS/VLF Code}

The original VHS/VLF code required substantial modification in order to simulate WTEs, due to the large bandwidth and almost impulsive nature of the triggering whistler signal. The original code employed global bandpass filters which can very easily cut off the ends of a ramped signal lying within the simulation zone.

Since the local wave frequency/wavenumber is now potentially a sharp function of $z$ and may vary substantially over the $z$ domain, the global filtering operation must be replaced by a matched filter. We first define a piecewise linear function $\Delta k(z)$, which is the average local wavenumber of the wavefield $\tilde{R}$. This function is derived by separately averaging $-\partial \phi / \partial z$ over 20 subsections of the $z$ grid. The range of the coordinate $V^{*}$ in the phase box, $V_{1}^{*} \rightarrow V_{2}^{*}$, is now made to be a function of $z$ and $t$. At each time step this range is updated and centered on the local resonance velocity given by

$$
V_{\text {res }}^{*}=\frac{V_{\mathrm{g}}-V_{\mathrm{res}}}{k_{0}} \Delta k(z)
$$

In order to effect the matched filtering operation at every time step we proceed as follows. The fast phase variation corresponding to $\Delta k(z)$ is first divided out of $\tilde{R}(z)$ to give field $\tilde{\tilde{R}}(z)$.

$$
\tilde{\tilde{R}}(z)=\tilde{R}(z) \mathrm{e}^{j \tilde{\Phi}(z)}
$$

where

$$
\tilde{\Phi}(z)=\int^{z} \Delta k\left(z^{\prime}\right) d z^{\prime}
$$

The field $\tilde{\tilde{R}}(z)$ is then spatially bandpass filtered to the range $-\Delta f_{\mathrm{m}}$ to $+\Delta f_{\mathrm{m}}$, where $\Delta f_{\mathrm{m}}$ is the desired frequency half bandwidth of the matched filter, usually $20-40 \mathrm{~Hz}$. The filtering operation in the code is per- 
formed by means of a spatial DFT/IDFT, and corresponds to a circular convolution with a sequence $h(z)$, that is,

$$
\tilde{\tilde{R}}^{\prime}(z)=\tilde{\tilde{R}}(z) * h(z)
$$

The fast phase variation is now restored giving a filtered signal $\tilde{R}^{\prime}(z)$

$$
\tilde{R}^{\prime}(z)=\tilde{\tilde{R}}^{\prime}(z) \mathrm{e}^{-j \tilde{\Phi}(z)}
$$

The resonant particle current $\tilde{J}$ is subjected to the same matched filtering operation in order to filter out immediately any HF noise generated by the discrete nature of the estimation process for resonant particle current $\tilde{J}$.

In addition to the matched filtcr, the code still cmploys a global bandpass filter with a substantially increased half bandwidth of $150 \mathrm{~Hz}$. This serves to prevent the function $\Delta k(z)$ from exceeding reasonable limits during the simulation. Care must be taken to ensure that this global filter does not clip the initiating whistler. This filter was turned off until the triggering process was completed.

\section{Results of the Computations}

The main philosophy behind these simulations has been to attempt to simulate the main categories of WTEs rather than attempt to reproduce specific observed events in detail. The reason for this is that although the $L$ shell and equatorial plasma density may be tolerably estimated from the SCALE facility, the ambient electron distribution function is to all intents and purposes unknown, as is the nature of the nonlinear loss process and hence the wave amplitude saturation level.

\section{Triggering of a Riser From the Top of a Whistler (Halley): Run A}

The first simulation demonstrates the triggering of a slow riser from the top of the observable whistler trace. The scenario is close to that of data examples 1 and 2 , in that the $L$ value assumed is 4.68 and the equatorial plasma density used in $203 \mathrm{~cm}^{-3}$, compared to a value of $190 \mathrm{~cm}^{-3}$ returned by the AVDAS SCALE facility. The code inputs a narrowband whistler waveform, with $(f, t)$ characteristics determined from the equations of Ho and Bernard [1973]. Parameters used for the input whistler segment are given in Table 3.

The ambient electron distribution is taken to consist of a cold plasma component plus a hot anisotropic component $F_{0}(\mu, W)$ with a functional form shown in (27) and plotted in Figure 2. The distribution function $F_{0}(\mu, W)$ is normalised to give a linear cyclotron growth rate at the equator of $180 \mathrm{~dB} \mathrm{~s}^{-1}$, measured at the base frequency of $3700 \mathrm{~Hz}$.

This run employs only one $V_{\perp}$ beam, with a pitch angle of $66^{\circ}$, which is where the contribution to $\tilde{J}$ is maximum. The initial whistler amplitude is taken to be
Table 3. Parameters for Input Whistler Segment: Runs A, B, and B1

\begin{tabular}{lr}
\hline \multicolumn{1}{c}{ Parameter } & \multicolumn{1}{c}{ Value } \\
\hline Initial frequency $f_{1}$ & $3700 \mathrm{~Hz}$ \\
Final frequency $f_{2}$ & $4200 \mathrm{~Hz}$ \\
A & 0.252 \\
Nose frequency $f_{n}$ & $3154 \mathrm{~Hz}$ \\
$f_{n} / f_{e}{ }^{a}$ & 0.377 \\
\hline
\end{tabular}

${ }^{a}$ Assuming a dipole field model and a low energy plasma in diffusive equilibrium.

$0.1 \mathrm{pT}$, and the saturation level is $6.8 \mathrm{pT}$, which allows a high degrec of nonlincarity. The internal matched filter has a half bandwidth of $32 \mathrm{~Hz}$, giving a whole bandwidth of about 1.5 trapping frequencies. The global filter has a half bandwidth of $50 \mathrm{~Hz}$ but is switched on only after triggering has taken place.

Figure 3a shows an $f-t$ spectrogram plot of the whole simulation. The initial whistler does not present very clearly in this plot since the time duration is very short $(44 \mathrm{~ms})$ and the bandwidth is high $(500 \mathrm{~Hz})$. The slow riser is triggered at $3900 \mathrm{~Hz}$, well before the top of the initial whistler segment. Once triggering of a large amplitude emission has occurred, the emission frequency is "grabbed" by the internal filter and the remainder of the whistler is filtered out.

The global bandwidth of the simulated emission corresponds to that of the global filter $(\sim 100 \mathrm{~Hz})$, but the emission has a noticeable gradient of frequency across the phase box, and so the local bandwidth is only about $40 \mathrm{~Hz}$ total. Considerable sideband structure will be noticed in the wavefield spectrum, which accords with the known fact that the nonlinear WPI system here is upper sideband unstable when the inhomogeneity is negative [Nunn, 1984]. The sweep rate of the triggered emission is quite low at about $150 \mathrm{~Hz} \mathrm{~s}^{-1}$, measured at the equator. Generally speaking, the emissions generated by this code have sweep rates on the low side compared with observations. It is not entirely clear why this is. It may be that if nonlinear 3D unducting processes are included in the simulation, the wavefield becomes more narrow band thus allowing a higher degree of nonlinearity and hence faster sweep rates.

Figure 4a shows an $f-t$ plot of the output time series $\tilde{R}$ emerging from the phase box on the receiver side. The frequency resolution of this plot is $8.56 \mathrm{~Hz}$, with a redundancy close to 6 . The field bandwidth is about $40 \mathrm{~Hz}$ total, and sideband structure is clearly apparent.

Figure 5a shows a time plot of wavefield amplitude emerging from the receiver side of the phase box. The received amplitude increases rapidly and exponentially to reach the saturation level, where it stays for the duration of the cmission.

Figure 6 a displays a snapshot of the field amplitude 

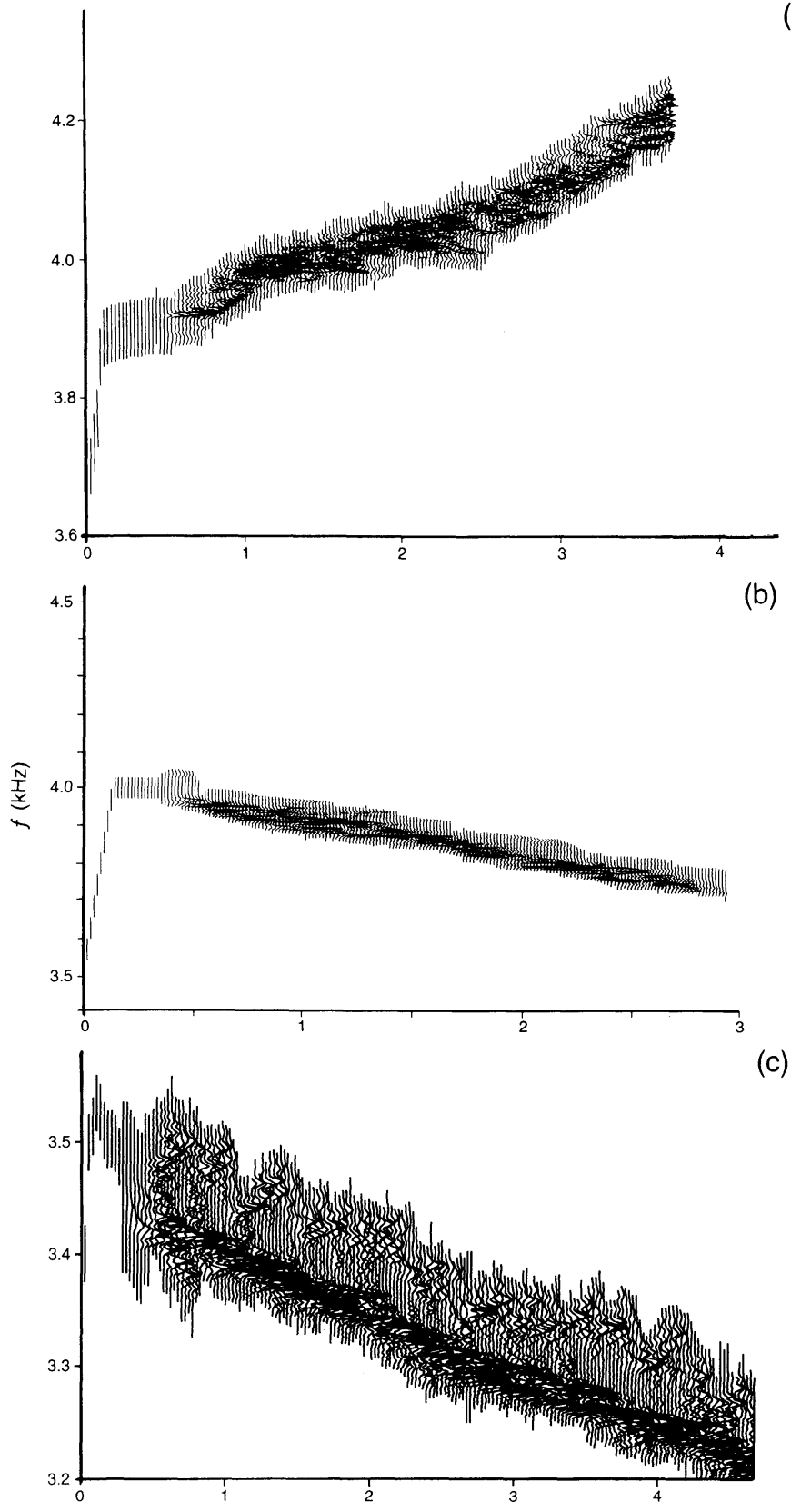

(b)

(c) (a)
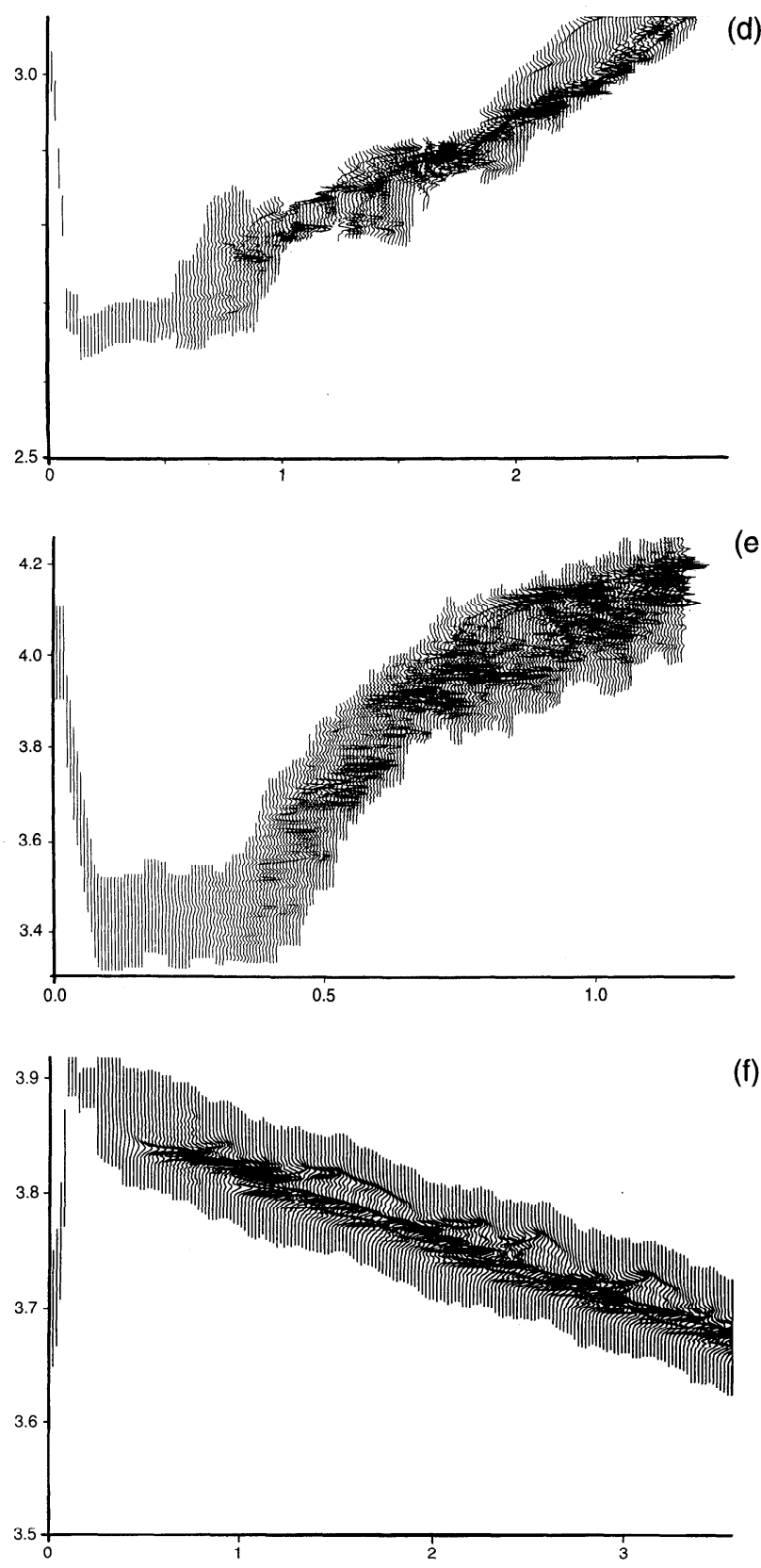

$t(\mathrm{~s})$

Figure 3. Spatial $f-t$ plots of the VLF/VHS simulation for different runs described in the text. (a) Run A: a slow riser triggered from the whistler upper arm at $L=4.67$. Each vertical line is a spatial DFT of the wavefield $R$ in the simulation box. (b) Run B: a slow faller triggered at $L=4.67$ from the upper arm of a whistler. The unperturbed distribution function is anisotropic at low pitch angles but close to isotropy at high pitch angles where particles are strongly nonlinear. (c) Run B1: a faller triggered at $L=4.67$. (d) Run C: a strong riser triggered from the lower arm of a whistler at $L=4.67$. (e) Run D: a strong riser triggered on the lower arm of a whistler at a lower latitude of $L=3.5$. (f) Run E: a slow faller triggered on the upper arm of a whistler at $L=4.67$. The simulation employs three $V \perp$ beams and has a low linear equatorial growth rate of $99 \mathrm{~dB} \mathrm{~s}^{-1}$.

$R(z)$ at a time $t=1869 \mathrm{~ms}$. This shows in essence the quasistatic generation region of a rising frequency emission. The sideband structure of the wavefield is evident in the periodic oscillations of amplitude. The location of the generation region (GR) is as expected theoreti- cally, with the low-amplitude end of the GR near the point of zero net inhomogeneity, which is on the receiver side of the equator for a riser [Helliwell, 1967].

Figure $7 \mathrm{a}$ shows $J_{r}$, and $J_{i}$ as functions of $z$ at time $t=1869 \mathrm{~ms}$. The oscillations due to the sidebands in 


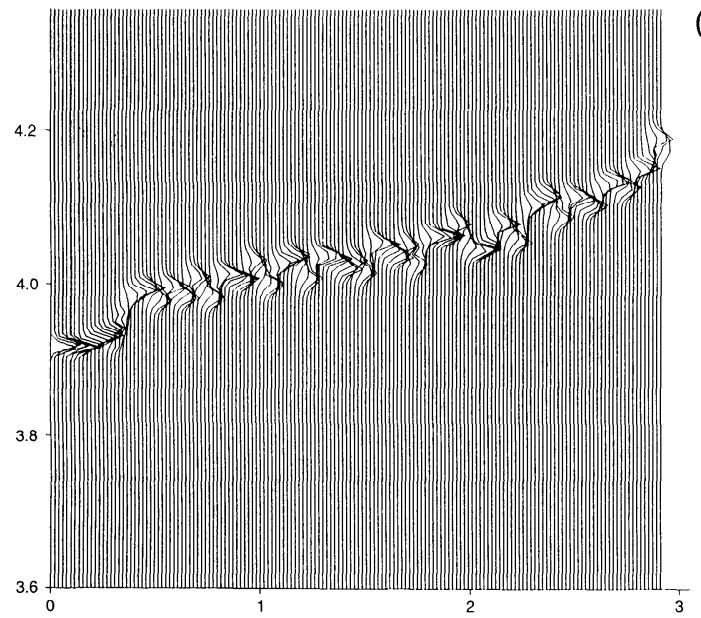

(a)

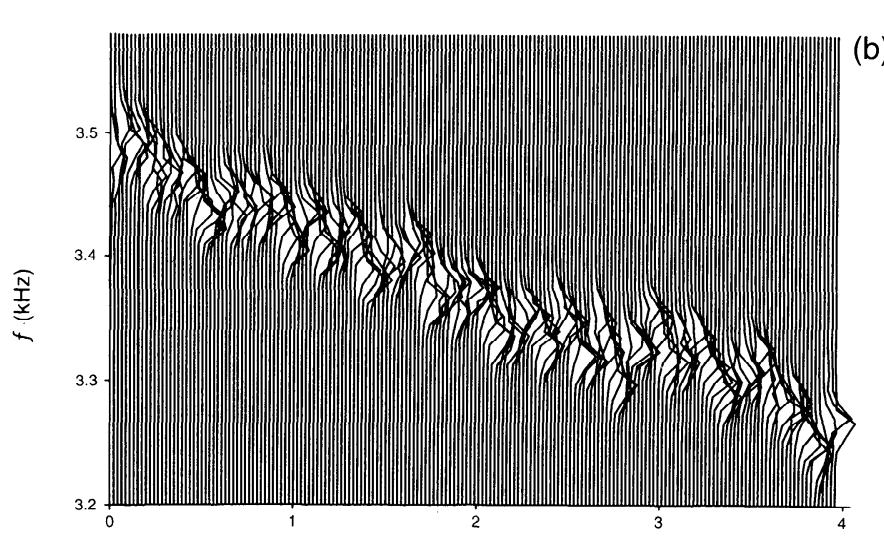

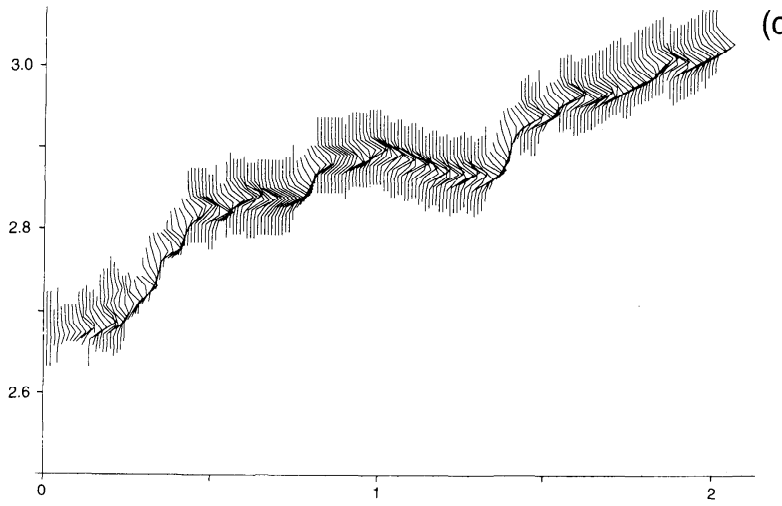

(c)

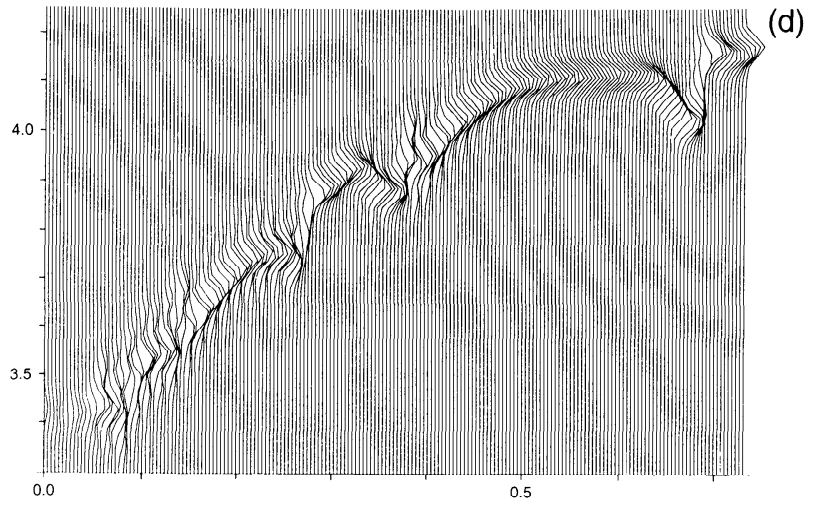

(e)

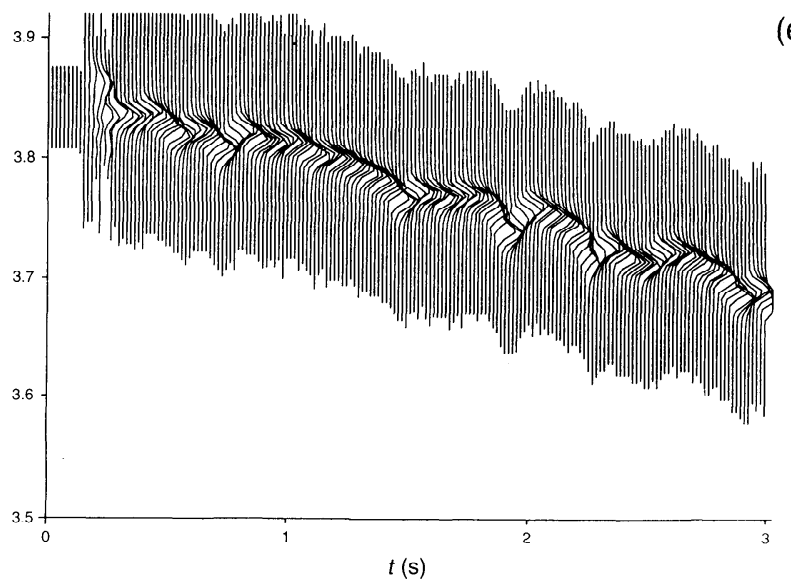

Figure 4. Frequency/time $f-t$ plots of the emission wavefield exiting from the simulation box for different runs. (a) Run A: the frequency resolution is $8.56 \mathrm{~Hz}$, redundancy equals 6 , and time resolution about $100 \mathrm{~ms}$. Not the structured form of the emission. (b) Run B1: the falling frequency emission, triggered at $L=4.67$. The frequency resolution is $11.55 \mathrm{~Hz}$. Note the marked structure (the emission seems to consist of a sequence of steeply falling elements). (c) Run C: the rising frequency emission at a resolution of $11.84 \mathrm{~Hz}$. The frequency sweep rate is fairly unstable with both rising and falling segments. (d) Run $D$ : the rising frequency emission at a resolution of $20 \mathrm{~Hz}$. The frequency sweep rate is fairly steep at $1 \mathrm{kHz} \mathrm{s}^{-1}$, good agreement with observations. (e) Run E: the triggered faller emission at a resolution of $5.62 \mathrm{~Hz}$.

$\tilde{R}$ are obvious. Apart from these, the form of $J_{r}$ and $J_{i}$ is entirely consistent with nonlinear trapping theory [Nunn, 1974], which predicts that the phase of $\tilde{J}$ is close to the phase locking angle $P_{0}$. Thus for $z>0 J_{r}$ and $J_{i}$ are both negative, and for $z<0, J_{r}$ is negative and $J_{i}$ positive. An interesting point is that in this simulation the wave envelope has extended well upstream of the equator, allowing significant trapping on the transmitter side of the equator. Not all simulations show this behavior. 


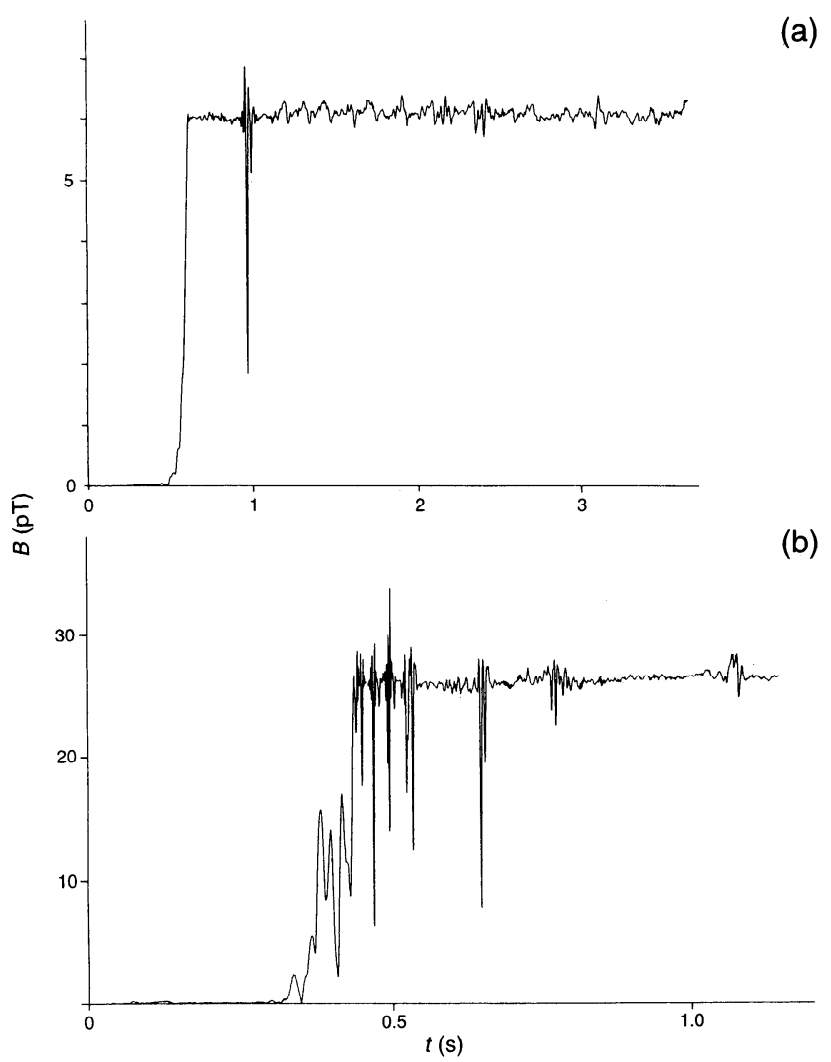

Figure 5. Exit amplitude as a function of time. Note the exponential growth phase, and then the leveling off at the saturation level. (a) Run A; (b) Run D.

\section{Triggering of Fallers From the Top of a Whistler at Halley: Runs B/B1}

The next two simulations are of fallers triggered from the top of the observable whistler trace. Generally, the simulation code tends to trigger risers preferentially. The main reasons for this are as follows. (1) $\Lambda$ ccording to Helliwell's [1967] phenomenological theory, riser gencration regions are located on the transmitter side of the equator, and faller generation regions are located on the receiver side. This is because zero net inhomogeneity is required at the low-amplitude end of the generation region in order for synchronism to be maintained between the wavefield and the nonlinear resonant current Nunn [1974]. The triggering pulse reaches the position for a rising frequency generation first. If conditions are right for triggering, a riser will be produced. Since the code is unable to simultaneously track the frequencies of both a riser and a faller, no faller will appear. (2) In the main body of the emission GR, $J_{i}$ is negative, which gives a positive contribution to $d f / d t$. The full equations governing wave phase development in time are given and discussed by Nunn [1990], and we shall not pursue this topic here.

It has been found that fallers may be triggered by the code under a number of circumstances as follows.

1. Weak linear growth rates and/or weak input pulses such that the GR becomes established on the receiver

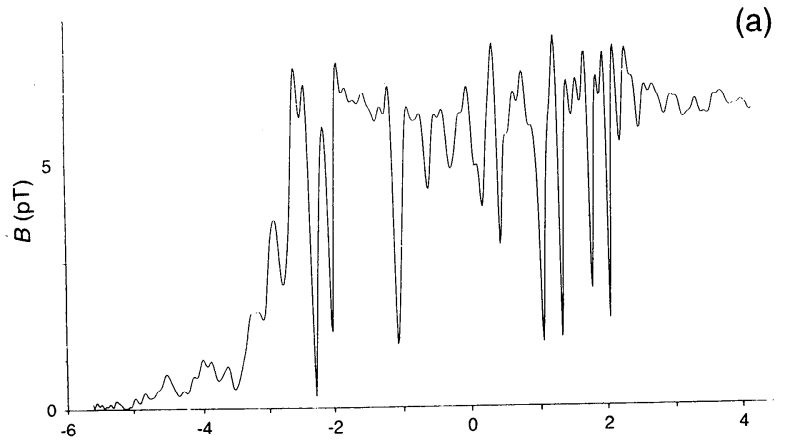

(a)
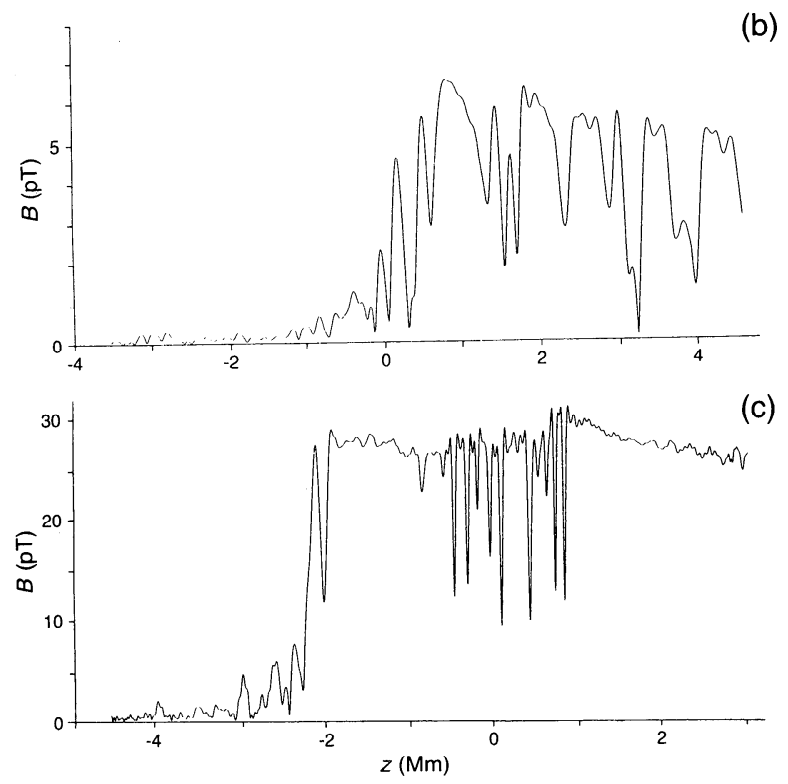

Figure 6. Plots of wavefield amplitude $R$ (in pT) against position $z$ at given times (representing snapshots of the generation region) for different runs. (a) Run A: $t=1869 \mathrm{~ms}$. (b) Run B: $t=708 \mathrm{~ms}$. The faller GR is, as expected, located on the receiver side of the equator. (c) Run D: $t=785 \mathrm{~ms}$. This shows the rising frequency generation region in the correct position with the low-amplitude end on the transmitter side of the equator.

side of the equator, which is the proper location for faller GR's [Helliwell, 1967]. In some cases where the linear growth rate is not weak enough, a faller may be triggered initially, but the GR then propagates upstream to give a riser. The resulting upward hook shaped emission is commonly observed experimentally with CW pulse triggering. Upward hooks are also fairly commonly triggered off the lower arm of the whistler (e.g., Figure 1g and are sometimes observed being triggered off the upper arm.

2. Fallers are readily simulated if an initial distribution function $F_{0}$ is selected which is anisotropic at low $\left(35^{\circ}-55^{\circ}\right.$ say) pitch angles, giving growth, but is close to isotropy at high pitch angles ( $>55^{\circ}$ say), giving damping. Essentially, the gradient term

$$
F_{0}^{\prime}=\left|\frac{\partial F_{0}}{\partial W}+\frac{2}{\omega_{0}} \frac{\partial F_{0}}{\partial \mu}\right|_{V_{z}=V_{\text {res }}}
$$

is positive for low $V_{\perp}$ and negative at high $V_{\perp}$. At large 


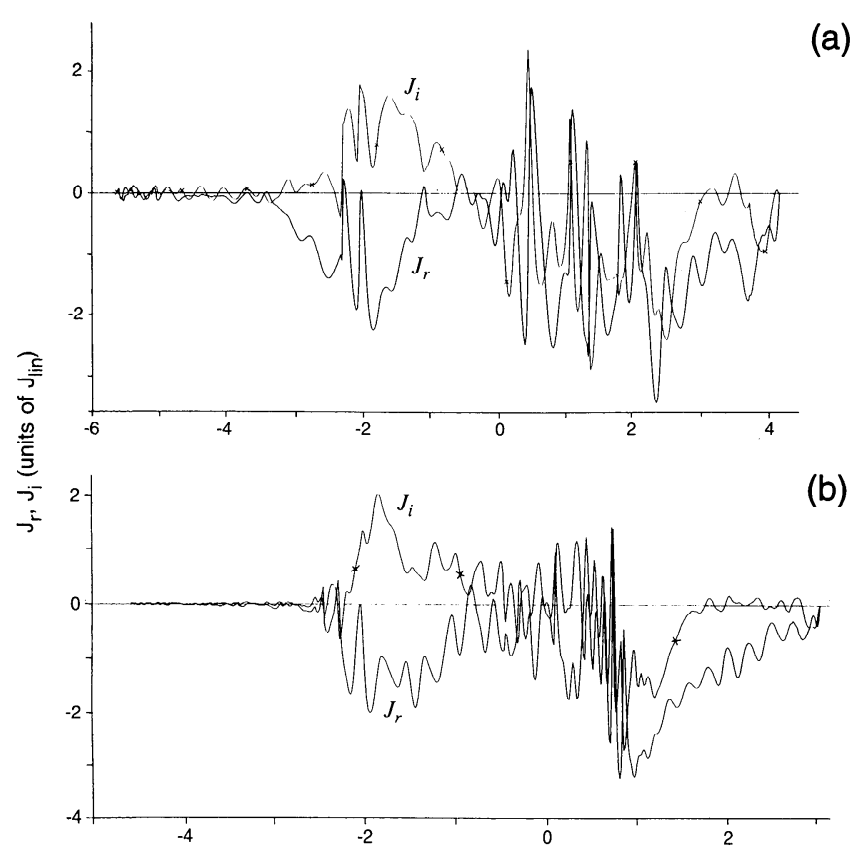

Figure 7. Plot of inphase and out-of-phase resonant particle currents $J_{r}$ and $J_{i}$ as functions of $z$ at given times for different runs. (a) Run A: $t=1869 \mathrm{~ms}$. Note (1) the prominent sideband structure and (2) the functional form indicative of nonlinear trapping in a parabolic inhomogeneity. (b) Run D: $t=785 \mathrm{~ms}$. The functional form is as expected for nonlinear trapping.

wave amplitudes where nonlinear trapping occurs, the highly nonlinear particles at high pitch angles give a net $J_{i}$ which is positive, which results in a consistent negative contribution to $d f / d t$.

The first example (run B) of a simulated triggered faller is for $L=4.67$ and an equatorial electron density of $203 \mathrm{~cm}^{-3}$, as in run A. The initiating whistler is also as in run A, taking the segment from 3500 to $4000 \mathrm{~Hz}$. In this run we do not define $F_{0}(\mu, W)$, but specify its gradient $F_{0}^{\prime}$ as a function of $V_{\perp}$ and equatorial $V_{z}$. The overall linear growth rate is low at $45 \mathrm{~dB} \mathrm{~s}^{-1}$. The code has a low pitch angle $\left(40^{\circ}\right)$ beam with positive $F_{0}^{\prime}$, giving a growth rate of $245 \mathrm{~dB} \mathrm{~s}^{-1}$. The second highly nonlinear beam has a pitch angle of $61^{\circ}$ and gives a linear damping of $-200 \mathrm{~dB} \mathrm{~s}^{-1}$. The initial whistler amplitude is weak at $0.1 \mathrm{pT}$, and the saturation level is taken to be $6 \mathrm{pT}$. The internal matched filter has a half bandwidth of $27 \mathrm{~Hz}$, and the global filter a semibandwidth of $50 \mathrm{~Hz}$. Figure $3 \mathrm{~b}$ shows the spatial $f-t$ plot for the whole simulation. A stable long enduring faller is produced with a sweep rate of about $-100 \mathrm{~Hz} \mathrm{~s}^{-1}$. This category of distribution function $F_{0}$ does not appear to give steep fallers with sweep rates $\sim 1 \mathrm{kHz} \mathrm{s}^{-1}$, as observed. Figure $6 \mathrm{~b}$ plots wave amplitude as a function of position at $t=708 \mathrm{~ms}$, and represents a snapshot of the faller generation region. As expected from theory, the GR is located on the receiver side of the equator.

The second example (run B1) of a simulated triggered faller is with the same $L$ value and electron density as in run A. The distribution function is low anisotropy and is exactly as in run $A$, as is the initial whistler segment. Input amplitude is weak at $0.1 \mathrm{pT}$, and the saturation level set at $6 \mathrm{pT}$. Linear growth rate is set high at $350 \mathrm{~dB} \mathrm{~s}^{-1}$ and the simulation uses only one $V_{\perp}$ beam. The internal matched filter has a half bandwidth of $29 \mathrm{~Hz}$, and the global filter a half bandwidth of $102 \mathrm{~Hz}$. Figure 8 shows a plot of average frequency in the simulation box against time. The initial $d f / d t$ is quite steep at $-350 \mathrm{~Hz} \mathrm{~s}^{-1}$, but this soon flattens to give a long enduring slow faller with a sweep rate of only $-50 \mathrm{~Hz} \mathrm{~s}^{-1}$. The spatial $f-t$ diagram for this run is shown in Figure 3c. Upper sideband activity is very marked. The $f-t$ plot of the field output sequence is shown in Figure $4 \mathrm{~b}$ with a frequency resolution of $11.55 \mathrm{~Hz}$. The emission is clearly structured and consists of a succession of steep falling elements, each with a sweep rate of about $-400 \mathrm{~Hz} \mathrm{~s}^{-1}$. It is not entirely clear why this run produces a faller. What seems to happen is the establishment of a faller GR on the receiver side of the equator as expected. As far as nonlinear particle dynamics is concerned, this is decoupled from the active region on the transmitter side of the equator by the strong sideband activity.

\section{Triggering of a Riser From the Lower Arm of a Whistler at Halley: Run C}

The code was next run using the lower segment of a whistler as the triggering signal. The assumed $L$ value was 4.67 as in run $\mathrm{A}$, with an equatorial electron density of $263 \mathrm{~cm}^{-3}$. The initial whistler was as in run A, except that the segment from 3000 to $2400 \mathrm{~Hz}$ only was taken. The unperturbed distribution function $F_{0}$ was exactly as in run $A$, except that the linear equatorial growth rate was taken to be $300 \mathrm{~dB} \mathrm{~s}^{-1}$, measured at $3000 \mathrm{~Hz}$. The linear growth rate at $2600 \mathrm{~Hz}$ where the triggering actually takes place is somewhat less than this. This run employs a single $V_{\perp}$ beam at a pitch angle of $59.3^{\circ}$. The internal matched filter has a half bandwidth of $32 \mathrm{~Hz}$, and the global filter a half bandwidth of $55 \mathrm{~Hz}$. Input

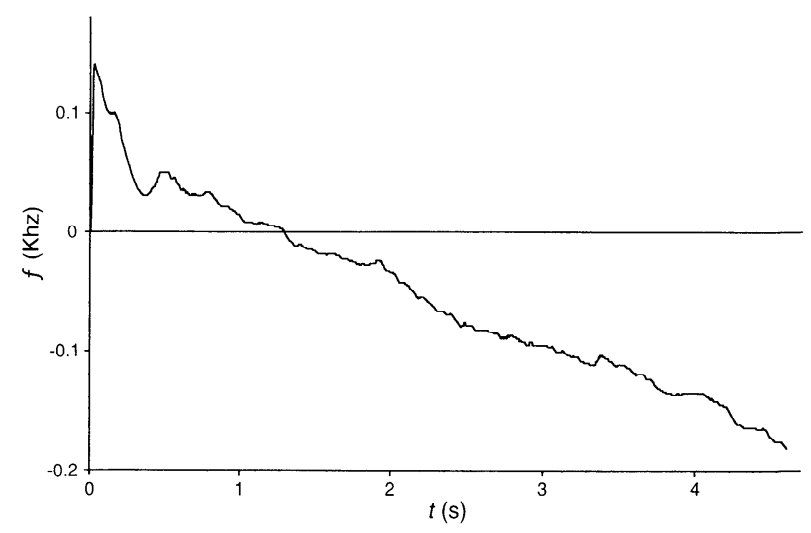

Figure 8. Run B1: Plot of average frequency against time for a faller triggered off the whistler upper arm at $L=4.67$. 
signal strength is taken to be $0.9 \mathrm{pT}$ and the saturation level is $6.5 \mathrm{pT}$.

Figure $3 \mathrm{~d}$ shows the spatial $f-t$ plot for the whole simulation. An emission is triggered at $2600 \mathrm{~Hz}$. The sweep rate is initially fairly flat, and then the emission becomes rather irregular, rising about $500 \mathrm{~Hz}$ in $2 \mathrm{~s}$. Figure $4 \mathrm{c}$ shows the $f-t$ diagram of the output sequence $\tilde{R}$ for the emission, using a frequency resolution of $11.48 \mathrm{~Hz}$. As expected the emission bandwidth is quite narrow, of order $30 \mathrm{~Hz}$. The emission generation region seems to be rather unstable, with a sweep rate that varies considcrably. One segment $0.25 \mathrm{~s}$ long has a swecp rate of $+700 \mathrm{~Hz} \mathrm{~s}^{-1}$, but there is a QCFE segment and also a falling frequency segment with a sweep rate of $-200 \mathrm{~Hz} \mathrm{~s}^{-1}$. The structure of the GR is similar to that of case A and will not be shown for reasons of space. In both cases there is a region of substantial wave amplitude on the transmitter side of the equator, where independent trapping occurs and where $J_{r}$ is negative and $J_{i}$ is positive. The unstable coupling between this interaction region and the one on the other side of the equator where $J_{i}$ is negative could be the cause of the dynamical instability in the GR.

\section{Triggering of a Riser From the Lower Arm of a Whistler at Faraday: Run D}

The next run aims to simulate the triggering of a steep riser from the lower arm of a whistler. Such emissions are produced at lower $L$ shells and often observed at Faraday station.

We take an $L$ value of 3.5 and an equatorial plasma density of $693 \mathrm{~cm}^{-3}$. The triggering whistler again uses the model of Ho and Bernard [1973] with a nose frequency now set at $8000 \mathrm{~Hz}$. The segment from 4000 to $3000 \mathrm{~Hz}$ is input into the simulation as the triggering signal. The unperturbed distribution function $F_{0}$ has the same form as in run $A$ but with a linear equatorial growth rate of $500 \mathrm{~dB} \mathrm{~s}^{-1}$ at $4 \mathrm{kHz}$. Initial signal strength is $8 \mathrm{pT}$, and the saturation amplitude is $30.5 \mathrm{pT}$. The simulation uses one $V_{\perp}$ beam with a pitch angle of $49.9^{\circ}$. The internal matched filter has a half bandwidth of $118 \mathrm{IIz}$, and the global filter has a half bandwidth of $413 \mathrm{~Hz}$.

Figure $3 \mathrm{e}$ shows the spatial $f-t$ plot for the whole simulation. A pronounced riser is triggered at $3350 \mathrm{~Hz}$. Figure $4 \mathrm{~d}$ shows the $f t$ plot for the output field, at a resolution of $20.38 \mathrm{~Hz}$. Emission bandwidth is about $120 \mathrm{~Hz}$ in total. After an initial flat region there follows a $300 \mathrm{~ms}$ riser with a frequency sweep rate of $+1 \mathrm{kHz} \mathrm{s}^{-1}$, which would be greater observed at the end of the field line. The sweep rate seems to ease off at higher frequencies where the growth rate is actually larger, and the emission ends with a small downward hook, but retriggers another riser.

Figure $6 \mathrm{c}$ represents a snapshot of the riser GR and is a plot of $R$ against $z$ at $785 \mathrm{~ms}$. This is the usual profile shape for a riser GR. Note the substantial side- band activity which seems to be confined to the equator. The lower curves on this graph are linear electrostatic growth rate and the fractional bandwidth that is Landau unstable. This data will not be utilised here. Figure $7 \mathrm{~b}$ plots currents $J_{r}$ and $J_{i}$ against $z$, also at $785 \mathrm{~ms}$. Strong nonlinear trapping is evident since the phase of $\tilde{J}$ is close to the local phase trapping angle. Figure $5 \mathrm{~b}$ shows the received wave amplitude $R$ as a function of time. Again note the nearly exponential increase followed by saturation at $27 \mathrm{pT}$.

\section{Triggering of a Faller From the Upper Arm of a Whistler at Halley (Simulation With Multiple $V_{\perp}$ Beams): Run E}

The last simulation to be presented is of a faller triggered from the upper arm of a whistler at $L=$ 4.67. The equatorial plasma density is assumed to be $200 \mathrm{~cm}^{-3}$, and the unperturbed distribution function is as in run $\mathrm{A}$. The triggering whistler is also as in run A, the segment from 3700 to $4200 \mathrm{~Hz}$ being injected into the simulation box. The input wave magnitude is set fairly high at $2.03 \mathrm{pT}$, and the saturation level is at $4.20 \mathrm{pT}$. In this run the wavefield is filtered to quite a narrow bandwidth, the half bandwidth of the matched filter being $14 \mathrm{~Hz}$, and the half bandwidth of the global filter bcing $70 \mathrm{~Hz}$.

This run employs three $V_{\perp}$ beams, with pitch angles of $51.9^{\circ}, 60.3^{\circ}$, and $66.6^{\circ}$. The linear equatorial growth rate is set low at $99 \mathrm{~dB} \mathrm{~s}^{-1}$ at the base frequency of $3700 \mathrm{~Hz}$, since low growth rates are known to favour the triggering of fallers. Figure $3 \mathrm{f}$ shows the spatial $f-t$ plot of the simulation. It is seen that a strong slow faller is triggered at about $3900 \mathrm{~Hz}$. The faller lasts $3 \mathrm{~s}$ and has a small sweep rate of about $-100 \mathrm{~Hz} \mathrm{~s}^{-1}$; Figure $4 \mathrm{e}$ shows the $f-t$ diagram of the emission with a frequency resolution of $5.62 \mathrm{~Hz}$. A curious structure now becomes apparent. The sweep rate varies substantially, and there are a number of short segments with sweep rates of $-400 \mathrm{~Hz} \mathrm{~s}^{-1}$.

\section{Conclusions}

Examination of BAS VLF data from IIalley and Faraday stations reveals many examples of whistler-triggered emissions. The main types of cvent quickly became apparent. For whistler-triggering at high $L$ values inside the plasmapause $(L=4.2-4.7)$ the dominant event type was the triggering of a slow riser or quasi-constant frequency emission from the top of the observable whistler trace. These emissions are sometimes very long enduring, up to several seconds long, and often exhibit some variability in frequency sweep rate along their length. Also commonly observed at high $L$ values are short steep fallers triggered off the top of the whistler trace. At lower $L$ shells ( $L=3.1-3.5$ ) triggering of emissions is generally less common. The normal emission type here is that of a steep riser triggered off the lower arm of 
the whistler trace, though upward hooks are sometimes also observed.

Simulations of these highly nonlinear phenomena were done with a 1D code assuming ducting/parallel propagation. Unfortunately, the 1D code is absolutely unstable when run with linear growth rates appropriate to the triggered emission problem, and nonlinear unducting losses have to be inserted phenomenologically in order to proceed with the simulation. The code uses the Vlasov VHS method, which is particularly well suited to this problem, since the parallcl velocity range in the phasc box is centred on the local resonance velocity in space and time. The simulations undertaken cannot purport to duplicate actual events precisely, because a number of inputs are unknown. These are (1) input wave amplitude at the entrance to the phase box; (2) linear growth rate; (3) unperturbed distribution function $F_{0}$; (4) the saturation level and the precise plasma dynamics of the saturation mechanism. Another area of difficulty is the choice for simulation bandwidth. This has been chosen to be of the order of or greater than the observed emission bandwidths. Unfortunately, the simulation spectrum tends to fill the available bandwidth, thus decreasing the level of nonlinearity in the WPI system. In the absence of filtering and nonlinear unducting loss the basic 1D VLF code gives, at large times, a wave output unlimited in both wave amplitude and bandwidth. It would appear that in the full 3D WPI problem in a duct the nonlinear processes act in such a way as to preserve spectral sharpness and the narrow band character of the emission.

The simulation of slow riscrs from the upper part of the whistler trace was easily achieved. These simulations were repeatable, stable and emissions were always produced provided that the linear growth rate exceeded a certain threshold in the region of $80 \mathrm{~dB} \mathrm{~s}^{-1}$, and that the initial pulse was able to reach nonlinear amplitudes by the time the equator was reached. Simulated emissions were very long enduring, longer than in reality. Sweep rates were in the right order of magnitude, $250 \mathrm{~Hz} \mathrm{~s}^{-1}$ being typical. The actual sweep rate obtained depends, in a rather complicated way, on linear growth rate, saturation amplitude, input amplitude and simulation bandwidth. Risers with steep sweep rates $\left(>1 \mathrm{kHz} \mathrm{s}^{-1}\right.$ at $L=4.7$ or $3 \mathrm{kHz} \mathrm{s}{ }^{-1}$ at $L=3.5$ ) are very difficult to simulate. The steepest obtained so far at $L=4.7$ is about $500 \mathrm{~Hz} \mathrm{~s}^{-1}$ in a long enduring WTE.

The code can be persuaded to produce triggered fallers, either by having a low linear growth rate or by employing a distribution function that is anisotropic at low pitch angles and isotropic at high (strongly nonlinear) pitch angles. Faller sweep rates wcre generally disappointingly low, about $-150 \mathrm{~Hz} \mathrm{~s}^{-1}$. Steep fallers with sweep rates $<-1 \mathrm{kHz} \mathrm{s}^{-1}$ have yet to be obtained. This must be an area for continuing effort. The code readily produces upward hooks with larger growth rates, when the initial faller GR works its way upstream and becomes a riser.

Triggering of risers from the lower whistler arm was very successful. Steeper sweep rates $\sim+1 \mathrm{kHz} \mathrm{s}^{-1}$ were obtained at $L-3.5$, in good agreement with observation, but very steep sweep rates $\sim 3 \mathrm{kHz} \mathrm{s}^{-1}$ have not been obtained as yet.

Regarding future plans, the code will shortly be migrated from the SUN Sparcserver to massively parallel machines. This will enable simulations to be made with higher bandwidth and more $V_{\perp}$ grid points. However, the results with many $V_{\perp}$ beams will look scarcely any different from the results presented here, and unless the distribution function $F_{0}$ is precisely known and defined there is hardly any point in having large numbers of $V_{\perp}$ beams. The real challenge for the future is to simulate the full 3D problem of nonlinear WPI in a cylindrical duct, but this is three orders of magnitude harder than the 1D problem. All things considered the 1D code has been very successful at simulating this highly complex nonlinear phenomenon.

Acknowledgments. The authors wish to acknowledge a grant from the Engineering and Physical Sciences Rescarch Council for computing resources on the Cray YMP at Rutherford Laboratory.

The Editor thanks Y. Omura and another referee for their assistance in evaluating this paper.

\section{References}

Brice, N., An explanation of triggered very-low-frequency emissions, J. Geophys. Res., 68, 4626, 1963.

Carlson, C. R., R. A. Helliwell, and U. S. Inan, Space-time evolution of whistler mode wave growth in the magnetosphere, J. Geophys. Res., 95, 15073, 1990.

Helliwell, R. A., Whistlers and Related Ionospheric Phenomena, Stanford Univ. Press, Stanford, Calif., 1965.

Helliwell, R. A., A theory of discrete VLF emissions from the magnetosphere, J. Geophys. Res., 72, 4773, 1967.

I Iclliwcll, R. $\Lambda$., Controlled stimulation of VLF emissions from Siple Station, Antarctica, Radio Sci., 18, 801, 1983.

Helliwell, R. A., and U. S. Inan, VLF wave growth and discrete emission triggering in the magnetosphere: A feedback model, J. Geophys. Res., 87, 3537, 1982.

Ho, D., and L. C. Bernard, A fast method to determine the nose frequency and minimum group delay of a whistler when the causative spheric is unknown, J. Atmos. Terr. Phys., 35, 881, 1973.

Laird, M. J., and D. Nunn, Full-wave VLF modes in a cylindrically symmetric enhancement of plasma density, Planet. Space Sci., 23, 1649, 1975.

Nunn, D., A self-consistent theory of triggered VLF emissions, Planet. Space Sci., 22, 349, 1974.

Nunn, D., A nonlinear theory of sideband stability in ducted whistler mode waves, Planet. Space Sci., 34, 429, 1984.

Nunn, D., The numerical simulation of VLF nonlinear waveparticle interactions in collision-free plasmas using the Vlasov hybrid simulation technique, Comput. Phys. Commun., 60, 1, 1990.

Nunn, D., A novel technique for the numerical simulation of hot collision-free plasma; Vlasov hybrid simulation, $J$. Comput. Phys., 108, 180, 1993. 
Omura, Y., and H. Matsumoto, Computer simulations of basic processes of coherent whistler wave-particle interactions in the magnetosphere, J. Geophys. Res., 87, 4435, 1982.

Omura, Y., and H. Matsumoto, Simulation study of frequency variations of VLF triggered emissions in a homogeneous field, J. Geomagn. Geoelectr., 37, 829, 1985.

Omura, Y., D. Nunn, H. Matsumoto, and M. J. Rycroft, A revicw of observational, theoretical and numerical studies of VLF' triggered emissions, J. Atmos. Terr. Phys., 53, $351,1991$.

Sá, L. A. D., A wave-particle-wave interaction mechanism as a cause of VLF triggered emissions, J. Geophys. Res., 95, 12277, 1990.

Sazhin, S., M. Hayakawa, and K. Bullough, Whistler diagnostics of magnetospheric parameters: A review, Ann. Geophys., 10, 293, 1992.

Smith, A. J., VELOX: A new VLF/ELF receiver in Antarctica for the Global Geospace Science mission, J. Atmos. Terr. Phys., 57, 507, 1995.

Smith, A. J., and K. H. Yearby, AVDAS-A microprocessorbased VLF signal processing and spectral analysis facility for Antarctica, Br. Antarct. Surv. Bull., 75, 1, 1987.

Smith, A. J., I. D. Smith, and K. Bullough, Methods of determining whistler nose frequency and minimum group delay, J. Atmos. Terr. Phys., 37, 1179, 1975.
Smith, A. J., D. L. Carpenter, and U. S. Inan, Whistlertriggered VLF noise bursts observed on the DE-1 satellite and simultaneously at Antarctic ground stations, Ann. Geophys., 3, 81, 1985.

Smith, A. J., P. Hughes, and K. H. Yearby, DSP-II and its applications: A unified approach to the acquisition and analysis of VLF radio wave data for research, RadioScientist, 5, 120, 1994.

Thorne, R. M., and Horne, R. B., Landau damping of magnetospherically reflected whistlers, J. Geophys. Res., 99, 17249, 1994.

Yearby, K. H., and A. J. Smith, The polarisation of whistlers received on the ground near $L=4, J$. Atmos. Terr. Phys., 56, 1499, 1994.

D. Nunn, Department of Electronics and Computer Science, University of Southampton, Southampton, SO17 1BJ, England. (e-mail: D.Nunn@ecs.soton.ac.uk)

A. J. Smith, British Antarctic Survey, High Cross, Madingley Road, Cambridge, CB3 0ET, England. (e-mail: A.J.Smith@bas.ac.uk)

(Received June 2, 1995; revised September 11, 1995; accepted October 12, 1995.) 